\title{
Equilibrium Exercise of European Warrants
}

\author{
Nikunj Kapadia ${ }^{1}$ \\ Gregory Willette
}

Original Version: March 2002

Current Version: October 2005

\footnotetext{
${ }^{1}$ Isenberg School of Management, University of Massachusetts. The paper has benefited from comments of N. Chidambaran, Hossein Kazemi, George Martin, Sanjay Nawalkha, N. R. Prabhala, Raghu Sundaram, seminar participants at Georgetown University, University of Maryland, and the University of Virgina. I thank Xiaoling $\mathrm{Pu}$ for computational assistance. The paper includes results that were previously circulated in a paper titled Dilution and the Valuation of Options and Non-Identical Warrants. Please address correspondence to Nikunj Kapadia, Isenberg School of Management, 121 Presidents Drive, University of Massachusetts, Amherst, MA 01003, 413-545-5643, or email: nkapadia@som.umass.edu.
} 


\section{Abstract \\ Equilibrium Exercise of European Warrants}

We develop a European warrant pricing theory that accounts for other securities in the capital structure of the firm, besides the stock and a warrant. An individual who owns a European warrant determines his exercise policy in equilibrium taking into account any transfer of wealth between stockholders and holders of other non-expiring securities. As a result, the equilibrium exercise policy differs from the call-like policy discussed in the literature. We demonstrate the dependence of the equilibrium exercise policy on the direction of wealth transfer. We derive the competitive equilibrium policy, and demonstrate how it can be computed and used for valuation. 
In developing a theory for the competitive equilibrium price of a warrant, Constantinides (1983) explicitly states that his results do not necessarily apply if there are wealth transfers between the stock- and warrant-holders and the other securities in the capital structure. ${ }^{1}$ In fact, this caveat applies to most of the existing literature on warrant pricing, in particular to Galai and Schneller's (1978) seminal result that the European warrant is a diluted call on the value of the firm. As a consequence, the result can be applied with certainty only if there are no other securities in the capital structure of the firm, severely limiting the application of the pricing theory to the valuation of warrants and other securities with embedded warrants, including convertible bonds and executive stock options.

In this paper, we develop a European warrant pricing theory to price warrants even when there are other securities in the capital structure of the firm. The point of departure from the standard model is that the European warrant-holder determines his exercise policy in equilibrium taking into account any transfer of wealth between stockholders and holders of other non-expiring securities. As a result, the equilibrium exercise policy differs from the calllike policy discussed in the literature, and depends on whether exercise increases or decreases the value of the other securities in the capital structure.

Although there exists a significant literature on the early exercise policy of the holder of an American warant as well as an important literature that examines exercise policies of holders of real options, ${ }^{2}$ there is little or no discussion regarding the equilibrium exercise policy for the European warrant. Given the existing literature, this is not entirely surprising. The equivalence of the European warrant to a call implies that the exercise policy of a call-holder whose actions have no impact on the stock price is identical to that of a warrant-holder whose exercise, in fact, impacts the stock price. We begin the paper by demonstrating that the strategic response of the warrant-holder coincides with that of a non-strategic investor when there are no other securities, besides a warrant and stock, in the capital structure of the firm. This is no longer the case under a more complex capital structure and, instead, we have to explicitly model the warrant-holder's exercise decision to derive the equilibrium policy.

\footnotetext{
${ }^{1}$ In footnote 10 of his paper, Constantinides (1984) provides the caveat that the results of the paper are under the assumption "...that the exercise of warrants does not redistribute wealth between senior bondholders and the other claimants to the firm, although it may redistribute wealth between the stockholders and the warrantholders." Because exercise of warrants impacts the firm, the exercise of warrants almost surely results in redistribution of wealth between the senior bondholders and the stock- and warrant-holders unless stock-holders can devise corporate policies to precisely offset the wealth transfer. Moreover, this caveat is not limited to senior debt, but also applies to any other security in the capital structure.

${ }^{2}$ Equilibrium early exercise policies for the American warrant are considered in Emanuel (1983), Constantinides (1984), Constantinides and Rosenthal (1984), and Spatt and Sterbenz (1988)). Ingersoll (1987) provides a lucid review of this literature. Exercise policies for holders of real options are considered in Williams (1993) and Grenadier (1996, 2002).
} 
In exercising the European warrant at maturity, a warrant-holder makes two related decisions. One, the warrant-holder decides the exercise threshold at which to start exercising the warrant. Two, he decides the number of warrants to exercise, knowing full well that any unexercised warrants will expire worthless. Both these decisions can be jointly formulated as being that of determining the warrant-holder's optimal exercise policy as a function of the underlying value of the firm, where the exercise policy is defined as the fraction of warrant-holding that is exercised at maturity. The optimal policy maximizes the value of the individual's own warrant-holding, after accounting for the effect of exercise on the stock and securities held by other individuals as well as the exercise policies of other individuals holding the warrant.

The presence of longer maturity securities - upstream securities that mature after the warrant - plays a critical role in determining the equilibrium policy. Depending on the firm's policy for the use of the exercise proceeds, exercise of warrants may result in an increase or decrease in the value of these securities. In the case of the former, an individual warrant-holder may partially exercise his warrant-holding, and let the remaining warrants expire worthless. The partial exercise policy is analogous to the sequential exercise of American warrants as first noted by Emanuel (1983) (although the latter does not necessarily result in non-exercised warrants expiring worthless). In the case of the latter, the equilibrium policy strictly precludes any exercise policy that does not result in all or none of the warrants being exercised. Such a block exercise policy has been defined and used to bound the American warrant price under a competitive equilibrium by Constantinides (1984).

Why is partial exercise an equilibrium policy? When upstream securities gain in value, the stock price is negatively impacted, hurting both existing shareholders and exercising warrantholders. The equilibrium response of a warrant-holder who does not benefit from the gain in value of upstream securities is to reduce the quantity exercised to mitigate the negative impact of his exercise. Consequently, his optimal policy may be to exercise only a fraction of his holdings.

How successfully warrant-holders can reduce wealth transfer to upstream securities depends on the concentration of warrant-holding. With greater competition between individual warrant-holders, warrant-holders are less effective in mitigating the negative impact of exercise. In fact, in a competitive equilibrium, competition between exercising warrant-holders reduces the payoff to the exercised warrant to zero over the range of firm values for which warrants are partially exercised. Any increase in firm value in this range solely benefits upstream securities.

Block exercise is an equilibrium policy for precisely the contrary reason. Here, exercise results in wealth transfer to the share- and warrant-holders, and the stock price increases 
with the amount of warrant that is exercised. Thus, the dominant strategy is to exercise all warrants at the lowest firm value where the warrant is not out-of-the-money. The block exercise equilibrium differs from both the partial exercise equilibrium and the option-like exercise policy as it strictly precludes partial exercise of warrants (the call and the textbook European warrant may be partially exercised in equilibrium). That is, the equilibrium policy is not convex-valued, and, therefore, the properties of the equilibrium prices are different. Specifically, at the exercise threshold, there are two equilibrium stock prices corresponding to the policy of exercising none or all, respectively, although the warrant price is zero in either of these equilibria. (In fact, the two equilibrium stock prices may be widely different - we provide an example where the stock price jumps by $14 \%$ on exercise). Finally, we also show that the block exercise is simply one example of an entire range of equilibrium policies where some - even if not necessarily all - partial exercise policies are excluded.

The perfectly competitive equilibrium policy is of both theoretical and practical importance as, noted by Spatt and Sterbenz (1988), warrants are often widely held across individuals. We derive and demonstrate how the competitive equilibrium policy can be computed. This is particularly important as the exercise policy must be determined before one can value the security. ${ }^{3}$ We demonstrate how the exercise policy can be used for valuation, and generalize the European warrant formula of Galai and Schneller (1978).

The existing literature for the American warrant has derived equilibrium exercise policies that are determined by wealth transfers only between warrant- and stock-holders. We complement this literature by focusing on the impact on equilibrium policies of the wealth transfers between warrant- and stock-holders and the non-stock securities in the capital structure. For example, partial exercise of American warrants is optimal as it transfers wealth from the shareholder to the exercising warrant-holder (Emanuel (1983)). Indeed, this observation has led to a discussion regarding how shareholders can prevent expropriation by a monopolizing warrantholder (Spatt and Sterbenz (1988)). In contrast, in our model, partial exercise occurs to reduce transfer of wealth to the other securities, and, therefore, if individuals holding different securities in the capital structure do not collude, shareholders benefit by allowing warrants to be monopolistically held on the exercise date. As noted in Constantinides (1983), the block exercise has been used in the literature to simplify American warrant pricing by imposing it as an artificial constraint (Ingersoll (1977), Brennan and Schwartz (1977)). In contrast, when there are other securities in the capital structure, we demonstrate that the block exercise policy is, in fact, an equilibrium policy and just one example of an entire range of non-convex equilibrium

\footnotetext{
${ }^{3}$ Our conclusion contrasts that of Constantinides (1984) in whose model there is no wealth transfer to other securities and the American warrant can be priced without explicit computation of the exercise policy.
} 
exercise policies.

Section 1 describes the setup of the model. Section 2 discusses the equilibrium exercise policy in the presence of longer-maturity securities. Section 3 applies the model to valuation. The last section concludes.

\section{The Model}

\subsection{Setup}

Consider a firm with 1 share and firm value per share of $V(t)>0$ at time $t \equiv T_{0}$ with $\alpha \geq 0$ European warrants $W$ of maturity $T_{1} \geq t$ and strike $X$ issued on its common stock $S$. Holders of the warrants have a right to purchase and create one share in the issuing firm. As we have normalized the number of shares to 1 , we can interpret $\alpha$ as the dilution factor of the warrant - the additional number of shares created by exercise of warrants for every original share. In addition to warrants of maturity $T_{1}$, the firm may have other outstanding securities (including warrants) of maturity greater than $T_{1}$. We designate the total time $t$ value of these securities as $\bar{W}(t)$. We will assume that the firm's dividend policy is known. As we only focus on European warrants, we may without loss of generality assume that the known ordinary dividends are zero (or, equivalently, define $V$ as the ex-dividend firm value). The firm may, however, pay extraordinary dividends from the proceeds of warrant exercise.

By the absence of arbitrage, for $t \leq T_{1}$,

$$
V(t)=S(t)+\alpha W(t)+\bar{W}(t)
$$

where $S$ is the stock price. As we focus on exercise strategies of expiring warrants at $T_{1}$ and because we do not model bankruptcy prior to this date, we do not model securities in the capital structure that mature on or before $T_{1}$. Equivalently, we may consider $V$ to be the firm value process less the value of any such securities. (We can relax the assumption that the warrants maturing at $T_{1}$ have the same strike.) For the illustrations in the paper, we will assume that unless there is a change in scale, $V(t)$ is lognormally distributed under the risk-neutral measure $Q$,

$$
d V(t)=r V(t) d t+\sigma V(t) d W^{Q}(t)
$$

where $r$ is the risk-free rate, $\sigma$ is the volatility, and $W^{Q}$ is the Wiener process.

Warrants may be held by a single or several individuals, each of whom optimally exercise 
their holding. Specifically, we assume that the warrant is held by a constant $K \geq 1$ individuals, indexed by $k=1,2, \ldots, K$, where each holder of the warrant holds none of the other securities. When $K=1$, a single monopolist holds all of the outstanding warrant (Emanuel (1983)). Our focus, however, will be the case of the perfectly competitive equilibrium, $K \rightarrow \infty$ (Constantinides (1984), Constantinides and Rosenthal (1984)). Denote $\alpha^{k}$ as the amount held by investor $k$ so that $\alpha=\sum_{k} \alpha^{k}$. We define each investor's exercise policy, $\delta^{k}\left(V\left(T_{1}\right)\right)$, as the fraction of $\alpha^{k}$ exercised at $T_{1}, 0 \leq \delta^{k} \leq 1$. The individual's equilibrium policy is $\delta^{k *}$. When $K>1$, we assume a Nash equilibrium where each investor optimally exercises $\delta^{k *}$ of $\alpha^{k}$ conditioned on other warrant holders exercising an optimal amount of their own holdings. Define $\delta\left(V\left(T_{1}\right) ; K\right)$ as the fraction of outstanding warrants exercised at equilibrium, $\delta=\left(\sum_{k} \delta^{k *} \alpha^{k}\right) /\left(\sum_{k} \alpha^{k}\right)$. We expect $\delta$ to be non-decreasing in $V\left(T_{1}\right)$.

Define $\bar{\alpha}(t) \equiv \bar{\alpha}(V(t)), t \leq T_{1}$, as the number of new shares created by exercise of warrants, $\bar{\alpha}(t)=\delta \alpha$, if $t=T_{1}$ and zero otherwise. Prices of all securities in the capital structure will depend on $V(t)$ and $\bar{\alpha}(t): W(t) \equiv W(V(t), \bar{\alpha}(t), t), S(t) \equiv S(V(t), \bar{\alpha}(t), t)$ and $\bar{W}(t) \equiv$ $\bar{W}(V(t), \bar{\alpha}(t), t)$. We assume that $W, S, \bar{W}$ are twice-differentiable in their first argument, and at least once-differentiable in their second and third arguments (every exercise policy $\delta^{k}$ ). This can be verified once $\bar{W}$ is parameterized. To focus on the effect of exercise by a warrant-holder, we will suppress other dependencies and write $S \equiv S\left(V, \delta^{k}(V)\right), \bar{W} \equiv \bar{W}\left(V, \delta^{k}(V)\right)$.

We assume that $0<\partial \bar{W} / \partial V<1$. The assumption that $\partial \bar{W} / \partial V \neq 0$ for all $V>0$ implies that $\bar{W}$ is risky. This assumption eliminates the trivial case when $\bar{W}$ is riskless debt, and, for most common securities, ensures that, in general, exercise of warrants affects the value of $\bar{W}$. To understand the usefulness of the upper bound on $\partial \bar{W} / \partial V$, observe that, for a given $S\left(T_{1}\right)$ and unobserved $V\left(T_{1}\right)$, equation (1) is a fixed-point problem. If $\partial \bar{W} / \partial V<1$, then for any observed stock price (and $\delta$ ), there is a unique $V$ by the contraction mapping theorem. This assumption is satisfied by many common securities in the capital structure. It can be easily verified to be true when $\bar{W}$ is a warrant of maturity $T_{2}>T_{1}$. It is also satisfied if $\bar{W}$ is risky debt as in Merton (1974). ${ }^{4}$

The payoff of the warrant, $W$, on exercise is

$$
W\left(T_{1}\right)=\max \left(S\left(T_{1}\right)-X, 0\right) .
$$

\footnotetext{
${ }^{4}$ The assumption imposes restrictions on the stochastic process followed by $V$. For instance, when $\bar{W}$ is a warrant of maturity $T_{2}>T_{1}$, the value of $\bar{W}$ on expiration of $W$ is equivalent to that of a diluted call written on $V$ (after suitable normalization for any extra shares created on exercise). Then to ensure that $\partial \bar{W} / \partial V<1$, we must have the delta of the call option be bounded by 1. Bergman, Grundy and Wiener (1996) provide the restrictions that must be imposed on $V$ for this to hold. For most standard models for $V(t)$ (lognormal, Heston's (1993) stochastic volatility model), the delta of the call is, in fact, bounded by 1.
} 
Non-exercised warrants expire worthless. The functional dependence of $W$ on $V\left(T_{1}\right)$ can be made explicit by substituting for $S\left(T_{1}\right)$ using equation (1).

We assume that the firm's policy regarding the use of the exercise proceeds is known. If the firm invests part of the exercise proceeds then, as in Constantinides (1984), the firm invests in zero-NPV projects. To illustrate the entire range of equilibrium exercise policies, it will be sufficient to assume that the firm invests in scale-enhancing projects or pays an extraordinary dividend. ${ }^{5}$ In such a case, on exercise of warrants at $T_{1}$, the number of outstanding shares increases from 1 to $1+\sum_{k=1}^{K} \delta^{k} \alpha^{k}$, and the total value of the firm can increase upto $\sum_{k=1}^{K} \delta^{k} \alpha^{k} X$ with the cash inflow from exercise, less the extraordinary dividend. To maintain the convention of normalizing the number of shares to 1 and using the superscript + to denote post-exercise values, let $V^{+}\left(T_{1}\right)$ be the value per share of the firm after exercise, and $D(V, \delta)$ be the extraordinary dividend per share. The relation between $V\left(T_{1}\right)$ (before exercise) and $V^{+}\left(T_{1}\right)$ (after exercise) is,

$$
V^{+}\left(T_{1}\right)=\frac{V\left(T_{1}\right)+\sum_{k=1}^{K} \delta^{k} \alpha^{k} X-D\left(V\left(T_{1}\right), \delta\right)}{1+\sum_{k=1}^{K} \delta^{k} \alpha^{k}} .
$$

\subsection{Equilibrium Exercise Strategies}

To ensure continuity with the existing literature on the American warrant, we define the following two sets of exercise strategies. First, define a block exercise policy as follows:

Definition 1 Block Exercise Policy: The equilibrium exercise policy $\delta^{k} \in\{0,1\}$ is a block policy if there exists a threshold $\bar{V}>0$ such that,

$$
\begin{array}{rlrl}
\delta^{k} & =1 & & \text { if } V\left(T_{1}\right) \geq \bar{V} \\
= & & \text { if } V\left(T_{1}\right) \leq \bar{V}
\end{array}
$$

The block policy specifically excludes the possibility that the warrant is partially exercised $\left(0<\delta^{k}<1\right)$.

Next, we define a partial exercise policy that allows for all $\delta^{k} \in[0,1]$.

\footnotetext{
${ }^{5}$ Alternative policies that have been discussed in the literature include investing in a riskfree asset and buying back stock (Spatt and Sterbenz (1988)). That firms invest at least some of the exercise proceeds in scale-enhancing projects is a common assumption (Ingersoll (1987)) and has empirical support. Empirical evidence in Mayers (1997) indicates that firms who issue convertible bonds undertake significant increases in capital expenditure around the period immediately after a conversion-forcing call.
} 
Definition 2 Partial Exercise Policy: The equilibrium exercise policy $\delta^{k}$ is a partial exercise policy if, for $V^{d} \leq V^{u}$ and $0 \leq \hat{\delta}^{k} \leq 1$,

$$
\begin{aligned}
\delta^{k} & =1 \quad \text { if } V\left(T_{1}\right)>\bar{V}^{u} \\
& =\hat{\delta}^{k}\left(V\left(T_{1}\right)\right) \quad \text { if } \bar{V}^{d} \leq V\left(T_{1}\right) \leq \bar{V}^{u} \\
& =0 \quad \text { if } V\left(T_{1}\right)<\bar{V}^{d}
\end{aligned}
$$

where $\hat{\delta}^{k}(V)$ is non-decreasing in $V$. An example of a partial exercise policy is the equilibrium exercise policy of the warrant-holder when $\bar{W} \equiv 0$. For this well-known special case, the exercise policy of the warrant-holder is identical to that of a call-holder: $V^{d}=V^{u}=X$, and at $V\left(T_{1}\right)=X$, every $\delta^{k} \in[0,1]$ is an equilibrium policy. More generally, we are interested in cases when $V^{d}<V^{u}$, and there is a non-trivial exercise policy where a fraction of outstanding warrant are exercised and the remainder expire worthless.

Both these policies have been discussed in the literature on the early-exercise of American warrants. Constantinides (1984) and Emanuel (1983) provide examples to demonstrate that the early-exercise policy is to partially exercise warrants over time (although this does not necessarily result in potentially in-the-money warrants expiring worthless). Constantinides (1984) compares the price of the American warrant to the price of a warrant where the space of exercise strategies is limited to the block exercise policy. However, as he notes, block exercise is an artificially imposed constraint, and not an equilibrium policy in the absence of the constraint. Although the block exercise is useful to contrast with the partial exercise policy, we will also demonstrate that the block exercise policy is simply one example of an equilibrium policy that is not convex-valued. In general, there is an entire range of similar equilibrium policies where some range of partial exercise polices are excluded (in essence, one may argue that the real distinction is between equilibrium policies that are convex-valued and not convex-valued).

\subsection{First Order Condition for Partial Exercise}

At $T_{1}$, the $k^{\prime}$ th investor who holds $\alpha^{k}$ of warrant $W$ chooses a exercise policy to maximize the value of his holding, $\delta^{k} \alpha^{k} W\left(T_{1}\right)$, given that other individuals also optimally exercise their holding, and subject to the constraint that $0 \leq \delta^{k} \leq 1$. We will defer the discussion of when this constraint is binding (i.e., $\delta^{k} \in\{0,1\}$ ) to the next section, and focus first on partial exercise. 
The first-order condition for partial exercise can be derived by observing that maximizing $\delta^{k} \alpha^{k} W\left(T_{1}\right)$ subject to (1) is equivalent to maximizing $V\left(T_{1}\right)-S\left(T_{1}\right)-\sum_{k^{\prime} \neq k} \delta^{k^{\prime} *} \alpha^{k^{\prime}}\left(S\left(T_{1}\right)-\right.$ $X)-\bar{W}\left(T_{1}\right)$. Differentiating the latter expression with respect to $\delta^{k}$, we get the first-order condition (FOC) as,

$$
\frac{\partial S\left(T_{1}\right)}{\partial \delta^{k}}\left(1+\sum_{k^{\prime} \neq k} \delta^{k^{\prime} *} \alpha^{k^{\prime}}\right)+\frac{\partial \bar{W}\left(T_{1}\right)}{\partial \delta^{k}}=0 .
$$

Equation (5) states that the warrant-holder makes his optimal decision by accounting for the price impact of exercise on the other securities in the capital structure.

Moreover, as $V\left(T_{1}\right)-S\left(T_{1}\right)-\sum_{k^{\prime} \neq k} \delta^{k^{\prime} *} \alpha^{k^{\prime}}\left(S\left(T_{1}\right)-X\right)-\bar{W}\left(T_{1}\right)=\delta^{k} \alpha^{k}\left(S\left(T_{1}\right)-X\right)$, we can also write $(5)$ as $\frac{\partial\left(\delta^{k} \alpha^{k}\left(S\left(T_{1}\right)-X\right)\right)}{\partial \delta^{k}}=0$. That is, the FOC also implies,

$$
\alpha^{k}\left(S\left(T_{1}\right)-X\right)+\delta^{k} \alpha^{k} \frac{\partial S\left(T_{1}\right)}{\partial \delta^{k}}=0
$$

From (3), the warrant is exercised only if $S\left(T_{1}\right)-X \geq 0$, so that when equation (6) is satisfied, $\partial S\left(T_{1}\right) / \partial \delta^{k} \leq 0$. The exercising warrant-holder trades off the gains from exercise (first term) against the change in the stock price that occurs on exercise (second term). A non-strategic warrant-holder, ignoring the price impact of his (and other individuals') exercise, will omit the second term in making his decision, exercise the warrant if it is in-the-money, and let it expire worthless otherwise. If $\bar{W} \equiv 0$ or $\partial \bar{W} / \partial \delta^{k}=0$, then equations (5) and (6) are satisfied with $\frac{\partial S\left(T_{1}\right)}{\partial \delta^{k}}=0$ and $S\left(T_{1}\right)=X$ (see equation (8) below).

\subsection{Strategic and Non-Strategic Exercise}

In existing literature, the warrant pricing problem has been solved for the special case when the capital structure consists of only the stock and a single warrant series and $\bar{W} \equiv 0$. Let $C(t) \equiv C(V(t), X ; T-t)$ be the value of a call of strike $X$ and maturity $T-t$. Then,

$$
W(t)=\frac{1}{1+\alpha} C(V(t), X ; T-t)
$$

An implication of this equivalence between the warrant and the call option is that the exercise policy of a warrant-holder is precisely the same as that of the call-holder. The following lemma explains why this is the case.

Lemma 1 If $\bar{W} \equiv 0$ or $\partial \bar{W} / \partial \delta^{k}=0$, the following statements are equivalent,

i. $\frac{\partial S\left(T_{1}\right)}{\partial \delta^{k}} \lesseqgtr 0$ 


\section{ii. $S\left(T_{1}\right) \gtreqless X$}

i.e., the optimization problem solved by a non-strategic investor who ignores the price impact of exercise is isomorphic to that of the strategic investor who does not ignore the price impact.

The equivalence of (i) and (ii) can be shown as follows. Differentiating equation (1) with respect to $\delta^{k}$, we get

$$
\frac{\partial S\left(T_{1}\right)}{\partial \delta^{k}}=\frac{-\alpha^{k}}{\left(1+\sum_{k^{\prime} \neq k} \delta^{k^{\prime} *} \alpha^{k^{\prime}}+\delta^{k} \alpha^{k}\right)}\left(S\left(T_{1}\right)-X\right)-\frac{1}{\left(1+\sum_{k^{\prime} \neq k} \delta^{k^{\prime} *} \alpha^{k^{\prime}}+\delta^{k} \alpha^{k}\right)} \frac{\partial \bar{W}}{\partial \delta^{k}},
$$

from which it immediately follows that, if $\bar{W} \equiv 0$ or $\partial \bar{W} / \partial \delta^{k}=0,\left(\partial S\left(T_{1}\right) / \partial \delta^{k} \lesseqgtr 0\right) \Leftrightarrow$ $\left(S\left(T_{1}\right)-X_{1} \gtreqless 0\right)$.

Lemma 1 explains why, when $\bar{W} \equiv 0$, the exercise policy of a strategic investor coincides with that of the non-strategic investor. From the FOC of equation (5), the strategic investor accounts for the impact of his exercise policy on the stock price. In contrast, a non-strategic individual ignores this price impact and instead follows a simpler rule of exercising all warrants when $S\left(T_{1}\right)>X$, and none when $S\left(T_{1}\right)<X$. Lemma 1 demonstrates that these two conditions are isomorphic, i.e., whenever $S\left(T_{1}\right)>X\left(S\left(T_{1}\right)<X\right)$, it is also true that $\frac{\partial S\left(T_{1}\right)}{\partial \delta^{k}}<0\left(\frac{\partial S\left(T_{1}\right)}{\partial \delta^{k}}>\right.$ $0)$. Moreover, $S\left(T_{1}\right)=X$ if and only if $\frac{\partial S\left(T_{1}\right)}{\partial \delta^{k}}=0$. In other words, when $\bar{W} \equiv 0$, the exercise policy for the non-strategic investor coincides with that of the strategic investor. Consequently, we can compute the correct equilibrium policy without explicitly evaluating (5) and considering the price impact of exercise. The equilibrium policy is to exercise all (none) of the warrant if it is in (out) of the money. When the warrant expires precisely at-the-money $\left(S\left(T_{1}\right)=X\right)$, every $\delta^{k} \in[0,1]$ is an equilibrium policy.

\section{Equilibrium Exercise Polices}

When there are longer-dated securities in the capital structure of the firm that are impacted by warrant exercise $\left(\partial \bar{W} / \partial \delta^{k} \neq 0\right)$, the warrant-holder will account for the change in the value of $\bar{W}$ in determining his exercise policy. The optimal exercise policy depends on whether exercise increases or decreases the value of $\bar{W}$. 


\subsection{Exercise Policy when $\partial \bar{W} / \partial \delta^{k}<0$}

The following lemma establishes the exercise policy for firm values where exercise decreases the value of $\bar{W}$.

Lemma 2 If $\partial \bar{W} / \partial \delta^{k}<0$ at $V\left(T_{1}\right)=\bar{V}$ and for all $\delta^{k} \in[0,1]$, then $\delta^{k *}(\bar{V}) \in\{0,1\}$.

If exercise of $W$ decreases the value of $\bar{W}$, then partial exercise cannot be an equilibrium policy. It is useful to explicitly consider why the above statement is true. We noted that the necessary conditions for partial exercise are equations (5) and (6). If both of these are not simultaneously satisfied, then we can rule out $\delta^{k *} \in(0,1)$. Equation (6) implies that $\alpha^{k}(S-X)+\delta^{k} \alpha^{k} \frac{\partial S}{\partial \delta^{k}}=0$ with $S-X \geq 0$ and $\partial S / \partial \delta^{k} \leq 0$. Thus, equations (5) and (6) can be simultaneously satisfied only if $\partial \bar{W} / \partial \delta^{k} \geq 0$. It follows that when $\partial \bar{W} / \partial \delta^{k}<0$, we get a corner solution, $\delta^{k *} \in\{0,1\}$. Intuitively, when exercise of the warrant decreases the value of upstream securities $\bar{W}$, there is a wealth transfer from $\bar{W}$ to the stock-holders and the exercising warrant-holders, and the stock price increases as more warrants are exercised. Thus, exercising all of an at- or in-the-money warrant gives the highest payoff to an individual warrant-holder.

Lemma 2 is useful as it suggests that a block exercise policy is likely to be the equilibrium strategy when $\partial \bar{W} / \partial \delta<0$, and that a partial equilibrium strategy can only be an equilibrium strategy when $\partial \bar{W} / \partial \delta>0$. Below, we derive a sufficient condition for a partial exercise equilibrium that requires $\partial \bar{W} / \partial \delta^{k}$ to be positive only in a certain range of firm values. We begin by providing a detailed illustration that provides the economic intuition underlying the importance of this condition for partial exercise. In the illustration, we assume $\bar{W}$ is a second warrant of maturity greater than $T_{1}$. We specifically choose this illustration of two tranches of warrants as it demonstrates a wide range of exercise strategies that are possible in equilibrium. ${ }^{6}$

\footnotetext{
${ }^{6}$ Two or more tranches of warrants are also common across international markets. In Schulz and Trautmann's (1994) empirical investigation of German warrants, more than half the firms in their sample have multiple series of warrants. In Japan, over the last decade, many companies have had multiple series of warrants or convertible bonds outstanding. For instance, in 1996, these firms included Daiwa Securities with 10, Nomura Securities with 7, Matsushita Electric with 4, and Sony Corporation with 5 issues (BZW Japanese Company Derivative Handbook, 1996.) The problem of valuing multiple tranches of warrant has been considered by Darsinos and Satchell (2002) and Dennis and Rendleman (2003). Neither of these papers model or consider the warrantholder's equilibrium exercise policy.
} 


\subsection{An Illustration: Strategic Equilibrium with Partial Exercise}

Let $\bar{W}$ be $\bar{\alpha}$ upstream warrants of maturity $T_{2}>T_{1}$ and strike $\bar{X}$. On exercise of $W$ at $T_{1}$, from (7), the value of $\bar{W}$ is,

$$
\bar{W}\left(T_{1}\right)=\frac{\bar{\alpha}}{1+\bar{\alpha}^{+}} C\left(V^{+}\left(T_{1}\right), \bar{X} ; T_{2}-T_{1}\right)
$$

where

$$
\bar{\alpha}^{+}=\frac{\bar{\alpha}}{\left(1+\sum_{k^{\prime}=1}^{K} \delta^{k^{\prime}} \alpha^{k^{\prime}}\right)}
$$

is the dilution factor for $\bar{W}$ adjusted for the new shares created at $T_{1}$ on exercise of $W$. In this section, we will assume that the firm does not pay any extraordinary dividend from the proceeds of exercise so that, from (4) with $D=0, V^{+}\left(T_{1}\right)=\left(V\left(T_{1}\right)+\sum_{k^{\prime}=1}^{K} \delta^{k^{\prime}} \alpha^{k^{\prime}} X\right) /\left(1+\sum_{k^{\prime}=1}^{K} \delta^{k^{\prime}} \alpha^{k^{\prime}}\right)$. (We will also, in a later section, separately consider the case when $D>0$.)

We will use equation (6) to understand the partial equilibrium policy. First, evaluate $\partial S\left(T_{1}\right) / \partial \delta^{k}$ by noting that conditioned on the $k^{\prime}$ th warrant-holder exercising $\delta^{k}, V\left(T_{1}\right)=$

$S\left(T_{1}\right)+\delta^{k} \alpha^{k}\left(S\left(T_{1}\right)-X\right)+\sum_{k^{\prime} \neq k} \delta^{k^{\prime}} \alpha^{k^{\prime}}\left(S\left(T_{1}\right)-X\right)+\bar{W}\left(T_{1}\right)$. Differentiating this expression with respect to $\delta^{k}$,

$$
\frac{\partial S\left(T_{1}\right)}{\partial \delta^{k}}=\frac{-\alpha^{k}\left(S\left(T_{1}\right)-X\right)}{1+\sum_{k^{\prime}=1}^{K} \delta^{k^{\prime}} \alpha^{k^{\prime}}}-\frac{1}{1+\sum_{k^{\prime}=1}^{K} \delta^{k^{\prime}} \alpha^{k^{\prime}}} \frac{\partial \bar{W}\left(T_{1}\right)}{\partial \delta^{k}} .
$$

In addition, by differentiating equation (9) we get,

$$
\frac{\partial \bar{W}\left(T_{1}\right)}{\partial \delta^{k}}=\left(\frac{\bar{\alpha}^{+}}{1+\bar{\alpha}^{+}}\right)\left(\frac{\alpha^{k}}{1+\sum_{k^{\prime}=1}^{K} \delta^{k^{\prime}} \alpha^{k^{\prime}}}\right)\left[\bar{W}\left(T_{1}\right)-\Delta\left(T_{1}\right)\left(V\left(T_{1}\right)-X_{1}\right)\right],
$$

where $\Delta\left(T_{1}\right)=\partial C\left(V^{+}\left(T_{1}\right), \bar{X} ; T_{2}-T_{1}\right) / \partial V^{+}\left(T_{1}\right)$ is the "delta" of the call. Note that $\partial \bar{W}\left(T_{1}\right) / \partial \delta^{k}$ is positive when $W$ is at- or close- to the money. From Lemma 2 , for a given firm value, there will not be partial exercise of warrants in equilibrium unless $\partial \bar{W}(T) / \partial \delta^{k}>0$. Not surprisingly, these are firm values where the warrant is close-to-the-money.

Substituting (11) and (12) into equation (6) and simplifying, we get,

$$
\frac{\partial\left(\delta^{k} \alpha^{k} W\left(T_{1}\right)\right)}{\partial \delta^{k}}=a_{1}\left(\delta^{k} ; K\right)\left(V\left(T_{1}\right)-X\right)-a_{2}\left(\delta^{k} ; K\right) \bar{W}\left(T_{1}\right)
$$


where

$$
\begin{aligned}
& a_{1}\left(\delta^{k} ; K\right)=\left(\frac{\alpha^{k}}{1+\sum_{k^{\prime}=1}^{K} \delta^{k^{\prime}} \alpha^{k^{\prime}}}\right)\left[1-\frac{\delta^{k} \alpha^{k}}{1+\sum_{k^{\prime}=1}^{K} \delta^{k^{\prime}} \alpha^{k^{\prime}}}+\left(\frac{\bar{\alpha}^{+}}{1+\bar{\alpha}^{+}}\right)\left(\frac{\delta^{k} \alpha^{k}}{1+\sum_{k^{\prime}=1}^{K} \delta^{k^{\prime}} \alpha^{k^{\prime}}}\right) \Delta\left(T_{1}\right)\right], \\
& a_{2}\left(\delta^{k} ; K\right)=\left(\frac{\alpha^{k}}{1+\sum_{k^{\prime}=1}^{K} \delta^{k^{\prime}} \alpha^{k^{\prime}}}\right)\left[1-\frac{\delta^{k} \alpha^{k}}{1+\sum_{k^{\prime}=1}^{K} \delta^{k^{\prime}} \alpha^{k^{\prime}}}+\left(\frac{\bar{\alpha}^{+}}{1+\bar{\alpha}^{+}}\right)\left(\frac{\delta^{k} \alpha^{k}}{1+\sum_{k^{\prime}=1}^{K} \delta^{k^{\prime}} \alpha_{1}^{k^{\prime}}}\right)\right] .
\end{aligned}
$$

If the inequality constraints are not binding and the optimal exercise policy for the $k$ 'th warrant-holder is a partial exercise policy, then we can compute it by solving $\frac{\partial\left(\delta^{k} \alpha^{k} W\left(T_{1}\right)\right)}{\partial \delta^{k}}=0$ using equation (13). ${ }^{7}$

Figure 1 plots best response correspondences when two identical individuals hold all of $W$, i.e., $K=2$ and $\alpha^{1}=\alpha^{2}$. The optimal policy response for each individual is graphed as a function of the other individual's policy. The equilibrium policy is given by the intersection of the two graphs. As both individuals are identical, the individual policies are similar. In the example graphed, the fraction of the total warrants exercised in equilibrium at a firm value of 112.81 is $\delta=0.3$. For firm values greater (less) than 112.81, more (less) warrants will be exercised. The equilibrium exercise policy is a partial equilibrium policy: there exists a range of firm values where each individual warrant-holder exercises a fraction of his holding.

Figure 2 plots $\delta$ as a function of $K$ for the symmetric equilibrium. As $K$ explicitly enters in equation (13), the exercise policy depends on the distribution of warrant-holding. For every $K$, exercise begins at a firm value of 111.12. The rate of exercise, however, differs across $K$. For example, when $K=100$, all warrants are exercised at a firm value of 113.91 (the exercise policy for $K>100$ is virtually identical to that for $K=100$ ). On the other hand, a monopolist would exercise less than $40 \%$ of his holding at this identical firm value. Correspondingly, the value of the warrant is considerably higher when the monopolist exercises it. At $V\left(T_{1}\right)=113.91$, the value of the warrant for the monopolist is $\$ 0.71$. In contrast, when $K=100$, the value of this warrant is about half a cent.

Why are more warrants partially exercised when $K$ is higher? Recall from our previous discussion that when $\partial \bar{W} / \partial \delta>0$, there is a wealth transfer from the stock-holders to the holders of the upstream securities, and the stock price is strictly decreasing in $\delta$. The partial

\footnotetext{
${ }^{7}$ It is important to verify that a solution of $\frac{\partial\left(\delta^{k} \alpha^{k} W\left(T_{1}\right)\right)}{\partial \delta^{k}}=0$ is indeed an equilibrium policy (i.e., to compare with the payoff for $\left.\delta^{k *} \in\{0,1\}\right)$. For the special case of a competitive equilibrium, Proposition 1 provides a simple sufficient condition for the existence of a partial equilibrium policy. In general, any of the following are possible: (i) there may not exist a partial exercise policy in equilibrium for any $V\left(T_{1}\right)$; (ii) some partial exercise strategies (that satisfy the equation $\frac{\partial\left(\delta^{k} \alpha^{k} W\left(T_{1}\right)\right)}{\partial \delta^{k}}=0$ ) may not be optimal as they are dominated by a policy of exercising all or none of the warrants; and (iii) a partial equilibrium policy that is an equilibrium policy may not be the sole equilibrium policy, and, in addition, there may also exist a non-partial equilibrium policy. We provide an example for each of these possibilities in Section 2.4.
} 


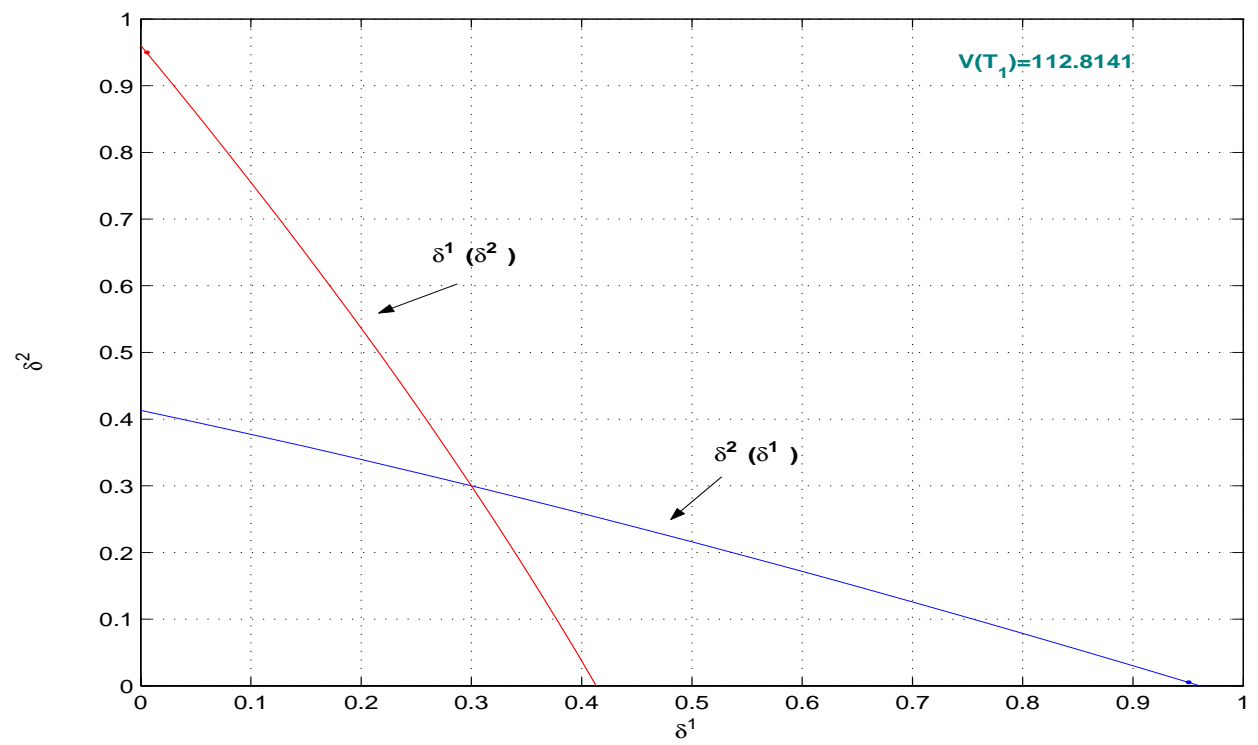

Figure 1: The graph plots the optimal exercise policy at maturity $T_{1}$ of one individual warrant-holder as a function of the the other warrant-holder's exercise policy, when two individuals equally hold the expiring warrant $W$, i.e., $K=2$. There are $\bar{\alpha}$ units of an upstream warrant $\bar{W}$ of strike $\bar{X}$ and maturity $T_{2}$. The parameters used to compute the exercise policy are: $\alpha=1, \bar{\alpha}=1, r=0, X=90, \bar{X}=100$, $T_{2}-T_{1}=5$, and $V\left(T_{1}\right)=112.8141$. The equilibrium exercise policy is given by the intersection of the two graphs, $\delta^{1 *}=\delta^{2 *}=0.30$.

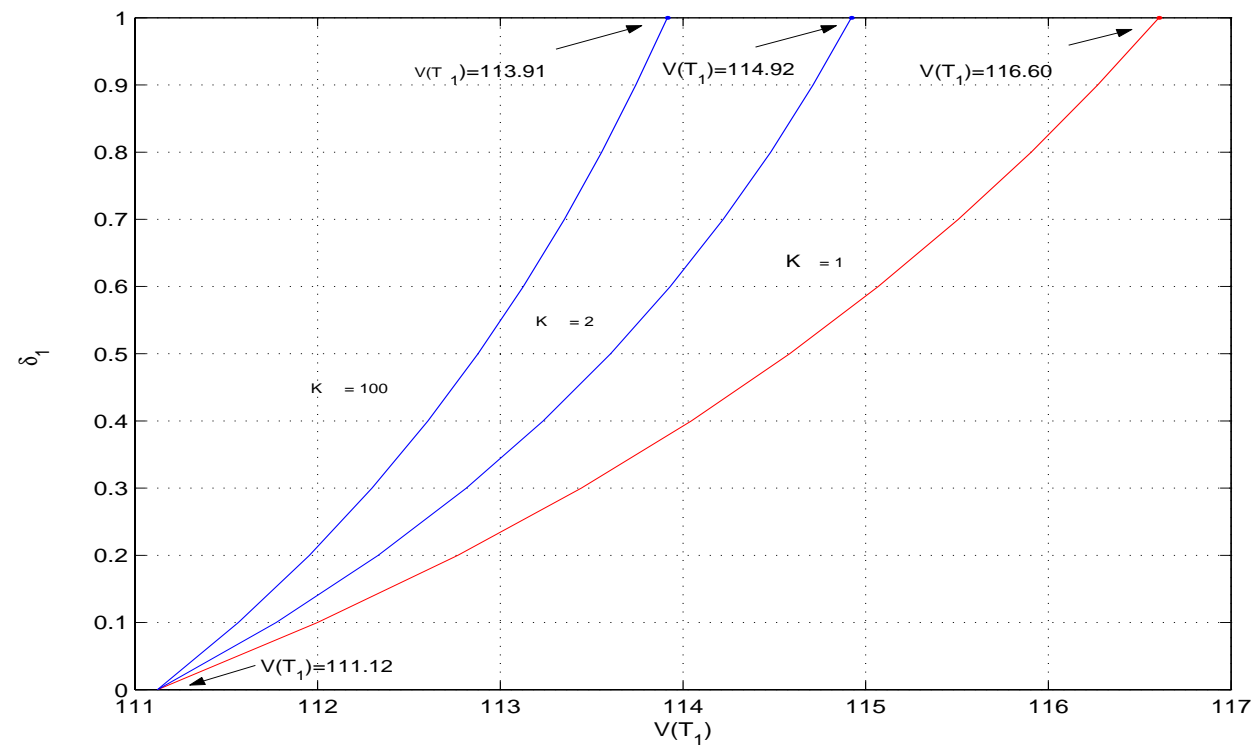

Figure 2: The graph plots the unique equilibrium exercise policy $\delta$ when $K$ individuals hold the expiring warrant $W$. The equilibrium is determined by a symmetric Nash equilibrium. There are $\alpha$ units of an upstream warrant $\bar{W}$ of strike $X$ and expiration date $T_{2}$. The parameters used to compute the exercise policy are: $\alpha=1, \bar{\alpha}=1, r=0, X=90, \bar{X}=100, T_{2}-T_{1}=5$. 
exercise of warrants is a response of the investor to reduce the magnitude of the wealth transfer by exercising fewer warrants. The magnitude of the wealth transfer is the least when each warrant is held by a single individual as he exercises the least of his holding. With higher $K$, the greater competition between individual warrant-holders leads, in equilibrium, to more warrants being exercised, a lower stock price, and lower payoff.

\subsection{Competitive Equilibrium with Partial Exercise}

In the illustration of the previous section, the fraction of warrants exercised in equilibrium, $\delta$, is a solution to a rather complicated fixed point problem that depends on $K$. It has been noted in the literature that warrants are often held by a large number of individuals (Spatt and Sterbenz (1988)). It is then of both theoretical and empirical importance to understand the exercise policy under a perfectly competitive equilibrium. The perfectly competitive equilibrium has been previously analyzed for the case of the single American warrant by Constantinides (1984) and Constantinides and Rosenthal (1984).

In a perfectly competitive equilibrium, let the warrant-holder take the price as given, ignoring any price impact of his own exercise. From equation (6), it is clear that, for a given stock price $S\left(T_{1}\right)$, the equilibrium policy is to exercise all of the warrant if $S\left(T_{1}\right)>X$, and none, if $S\left(T_{1}\right)<X$. Thus, the interesting case is for firm values where $S\left(T_{1}\right)=X$. We begin by defining this set of firm values. Let $\mathbf{V}$ be the set of firm values at $T_{1}$ defined by,

$$
\mathbf{V}=\{V \mid V=X+\bar{W}(V, \delta(V)), \delta \in[0,1]\}
$$

This set of firm values is defined by substituting $S(T)=X$ in equation (1). When $\partial \bar{W} / \partial V<1$, the set is non-empty, and, moreover, corresponding to each $\delta \in[0,1]$, there is a unique $V$.

The first part of the Proposition below states the exercise policy when $\frac{\partial \bar{W}}{\partial \delta}>0$.

Proposition 1 Part A: If $\frac{\partial \bar{W}}{\partial \delta}>0$ for $V \in \mathbf{V}$ and for all $\delta \in[0,1]$, then there exists a perfectly competitive partial equilibrium policy defined by $S(V, \hat{\delta}(V))=X$. That is,

i. There exists a $V^{d}$ such that when $V\left(T_{1}\right)<V^{d}, S\left(T_{1}\right)<X$ for all $\delta \in[0,1]$;

ii. There exists a $V^{u}$ such that when $V\left(T_{1}\right)>V^{u}, S\left(T_{1}\right)>X$ for all $\delta \in[0,1]$;

iii. On the interval $\left[V^{d}, V^{u}\right]$, there is a unique continuous increasing function $\hat{\delta}(V)$ defined by $S(V, \hat{\delta}(V))=X$, such that

A. $\hat{\delta}\left(V^{d}\right)=0$, 
B. $\hat{\delta}\left(V^{u}\right)=1$,

C. for $0 \leq \delta<\hat{\delta}(V), S(V, \delta)>X$, and

D. for $1 \geq \delta>\hat{\delta}(V), S(V, \delta)<X$.

Proof of Proposition 1: See Appendix.

Proposition 1 defines and demonstrates the existence of a stable competitive equilibrium. In addition, the proposition precisely identifies the number of warrants exercised in equilibrium. The equilibrium consists of two thresholds, $V^{u}$ and $V^{d}$. When $V\left(T_{1}\right) \geq V^{u}\left(V\left(T_{1}\right) \leq V^{d}\right), \delta$ is equal to $1(0)$. When $V^{d}<V\left(T_{1}\right)<V^{u}$, warrants are partially exercised in equilibrium, and the precise number exercised, $\hat{\delta}(V)$, is uniquely defined by $S(V, \hat{\delta}(V))=X$. In other words, for all $V\left(T_{1}\right) \in\left[V^{d}, V^{u}\right]$, just a sufficient number of warrants are exercised to make the payoff at exercise zero. In the absence of collusion, no individual warrant holder is able to profitably exercise $W$ in the interval $\left[V^{d}, V^{u}\right]$. The stock price remains constant at $X$ over all this range of firm values, and the value of both exercised and unexercised warrants is equivalently zero. In his analysis of the competitive equilibrium for the early exercise of an American warrant, Constantinides (1984) also demonstrates an equilibrium where the value of an exercised warrant is equal to that of an unexercised warrant.

To illustrate, consider the example discussed in the previous section. Write the necessary condition of equation (13), $\partial\left(\delta^{k} \alpha^{k} W\left(T_{1}\right)\right) / \partial \delta^{k}=0$, as $V\left(T_{1}\right)=X+\frac{a_{2}}{a_{1}} \bar{W}\left(T_{1}\right)$. Assume a symmetric equilibrium, $\alpha^{k}=\alpha / K$. Then, taking the limit $K \rightarrow \infty$ reduces the first-order condition to,

$$
V\left(T_{1}\right)=X+\bar{W}\left(T_{1}\right)
$$

which is, of course, the set of firm values corresponding to $\mathbf{V}$. For the competitive equilibrium to exist, there must exist a non-decreasing exercise policy $\hat{\delta}\left(V\left(T_{1}\right)\right)$ for which equation (15) is satisfied for every value of $\hat{\delta} \in[0,1]$. The computation of the competitive equilibrium policy, $\hat{\delta}\left(V\left(T_{1}\right)\right)$, can be viewed as the inverse problem of solving for $V\left(T_{1}\right)$ for a given $\hat{\delta} \in[0,1]$. As there is one-to-one mapping between $\hat{\delta}$ and $V$, each value of $\hat{\delta} \in[0,1]$ corresponds to a firm value in $\left[V^{d}, V^{u}\right]$, with $V^{d}$ and $V^{u}$ as the firm values corresponding to $\hat{\delta}=0$ and $\hat{\delta}=1$, respectively. The unique mapping from $\hat{\delta}$ to $V$ is assured because $\partial \bar{W} / \partial V=\frac{\bar{\alpha}}{1+\bar{\alpha}^{+}} \partial C / \partial V<1$. The existence of the unique competitive equilibrium is guaranteed if, in addition, $\partial \bar{W} / \partial \hat{\delta}>0$ 
for $V \in \mathbf{V}$ and for all $\delta \in[0,1]{ }^{8}$ To demonstrate this, note that we can write the inverse problem of $(15)$ as $V(\hat{\delta})-X=\bar{W}(V(\hat{\delta}), \hat{\delta})$. Differentiating with respect to $V$ and applying the Implicit Function theorem, $d V / d \hat{\delta}=(\partial \bar{W} / \partial \hat{\delta}) /(1-\partial \bar{W} / \partial V)$, and is positive if $\partial \bar{W} / \partial \hat{\delta}>0$. An application of the Inverse Function Theorem then establishes the existence of the competitive partial exercise policy $\hat{\delta}(V)$.

The proposition is especially useful as it demonstrates that the competitive equilibrium policy can be computed in a straightforward manner. This is particularly fortunate as the assumption that warrants are widely held is likely to be descriptive of actual warrant holding as noted by Spatt and Sterbenz (1988). For $K<\infty$, the equilibrium exercise policy must simultaneously satisfy each individual's first-order condition, and the computation of this policy is a complicated fixed-point problem. Here, the perfectly competitive policy may also serve as a close approximation. It has been noted that with increasing competition, the convergence to a competitive equilibrium is rapid. Grenadier (2002) provides an example where the value of an option to postpone investment reduces rapidly with competition with the option premium reducing to almost zero for 100 individuals (see his equation (32)). In the illustration of the previous section, the exercise policy for $K>>100$ is virtually identical to that of $K=100$.

The analysis also demonstrates how the exercise policy impacts the securities in the capital structure. As we noted, for $V\left(T_{1}\right) \in\left[V^{d}, V^{u}\right]$, the stock price has zero volatility and remains fixed at $X$. Therefore, any change in firm value within this range results in an identical change in $\bar{W}$, irrespective of the specific securities that make up $\bar{W}$.

Finally, the call-like exercise policy may be considered a special case of the competitive equilibrium when $\bar{W}=0$. Consider the set of values defined by $\mathbf{V}$ in (14). Substituting $\bar{W}=0$, observe that there is only one firm value defined by $S(V, \delta)=X$, i.e., $V=X$. This suggests that partial exercise of warrants may occur in equilibrium only at $V\left(T_{1}\right)=X$. In fact, this precisely defines the exercise policy of the single warrant as every $\delta \in[0,1]$ is an equilibrium partial exercise policy at this firm value. Lemma 1 provides the economic intuition for this. When $\bar{W} \equiv 0$, every investor, irrespective of his holding, behaves as if the stock price is independent of his exercise, and the equilibrium policy under the assumption that warrants are held by non-strategic naive investors replicates that of the competitive equilibrium.

\footnotetext{
${ }^{8}$ Write $\partial \bar{W}\left(T_{1}\right) / \partial \delta^{k}$ in equation (12) as

$$
\left(\frac{\bar{\alpha}^{+}}{1+\bar{\alpha}^{+}}\right)\left(\frac{\alpha^{k}}{1+\sum_{k^{\prime}} \delta^{k^{\prime}} \alpha^{k^{\prime}}}\right)\left[\left(1-\Delta\left(T_{1}\right)\right) \bar{W}\left(T_{1}\right)-\Delta\left(T_{1}\right)\left(1+\sum_{k^{\prime}=1}^{K} \delta^{k^{\prime}} \alpha^{k^{\prime}}\right)\left(S\left(T_{1}\right)-X\right)\right] .
$$
}

It follows that at $S\left(T_{1}\right)=X, \partial \bar{W}\left(T_{1}\right) / \partial \delta^{k}>0$, i.e., $\bar{W}$ is increasing in $\delta^{k}$ when $W$ is exercised at firm values where it is precisely at-the-money $(V \in \mathbf{V})$. 


\subsection{Competitive Equilibrium with Block Exercise}

Part B of Proposition 1 gives the block exercise threshold when $\partial \bar{W} / \partial \delta^{k}$ is negative.

Proposition 1 Part B: If $\partial \bar{W} / \partial \delta<0$ for $V \in \mathbf{V}$ and for all $\delta \in[0,1]$, then there exists an exercise threshold $\bar{V}$ defined by $S(\bar{V}, 1)=X$ such that,

$$
\begin{aligned}
& \text { i. For } V\left(T_{1}\right) \geq \bar{V}, S(V, 1) \geq X\left(\text { for } V\left(T_{1}\right)>\bar{V}, S(V, 1)>X\right) \text {. } \\
& \text { ii. For } V\left(T_{1}\right)=\bar{V}, S(V, \delta)<X \text { for } \delta \in[0,1) . \\
& \text { iii. For } V\left(T_{1}\right)<\bar{V}, S(V, \delta)<X \text { for } \delta \in[0,1] \text {. }
\end{aligned}
$$

The exercise threshold is defined by substituting $S=X$ and $\delta=1$ in equation (1), $\bar{V}=$ $X+\bar{W}(\bar{V}, 1)$, as the lowest firm value at which all warrants may be exercised. The option is out-of-the-money for all firm values below $\bar{V}$. At $V\left(T_{1}\right)=\bar{V}$, both $\delta=1$ and $\delta=0$ are equilibrium strategies. That is, if warrant-holders exercise, they exercise all their warrants. At $\delta=1$, the stock price is precisely equal to the strike, and at $\delta=0$, strictly less than the strike. Whether or not warrant-holders exercise, the warrant price is zero; however, the prices of the stock (and the other securities) may differ widely. As a consequence, the characteristics of equilibrium prices in the block equilibrium differ from those in the partial exercise equilibrium. In the competitive partial exercise equilibrium, the stock price has zero volatility for firm values corresponding to $S\left(T_{1}\right)=X$. In the block equilibrium, on exercise, the stock price at $\bar{V}$ jumps to $X$ from a level strictly lower than $X .^{9}$

The block exercise is an equilibrium policy that is not convex-valued. In fact, there is an entire class of similar equilibrium policies. In both parts of the Proposition, we assumed that $\partial \bar{W} / \partial \delta$ is positive or negative for every $\delta \in[0,1]$. This may not be the case. Instead, $\partial \bar{W} / \partial \delta$ may be positive for $\delta \in\left[\delta^{d}, 1\right]$, where $0<\delta^{d} \leq 1$, and negative otherwise. As Part A of Proposition 1 also holds if we limit its application to this range of $\delta$ (the identical proof applies for $\delta \in\left[\delta^{d}, 1\right]$ instead of $\left.\delta \in[0,1]\right)$, there will be partial exercise in equilibrium over a range of firm values $\left[V^{d}, V^{u}\right]$, where $\delta\left(V^{d}\right)=\delta^{d}$ and $\delta\left(V^{u}\right)=1$. We may also expect, from Part B of the Proposition and as we illustrate below, that exercising none of the warrants will also be an equilibrium policy at $V^{d}$. This results in additional equilibrium policies that are not convex-valued.

To illustrate, we return to our example with one modification. We now assume that the

\footnotetext{
${ }^{9}$ The call-like exercise strategy of the warrant when $\bar{W} \equiv 0$ differs from the partial exercise equilibrium in that the stock price is equal to $X$ at only one firm value $\left(V\left(T_{1}\right)=X\right)$, and from the block exercise in that the stock price at the exercise threshold is independent of $\delta$.
} 


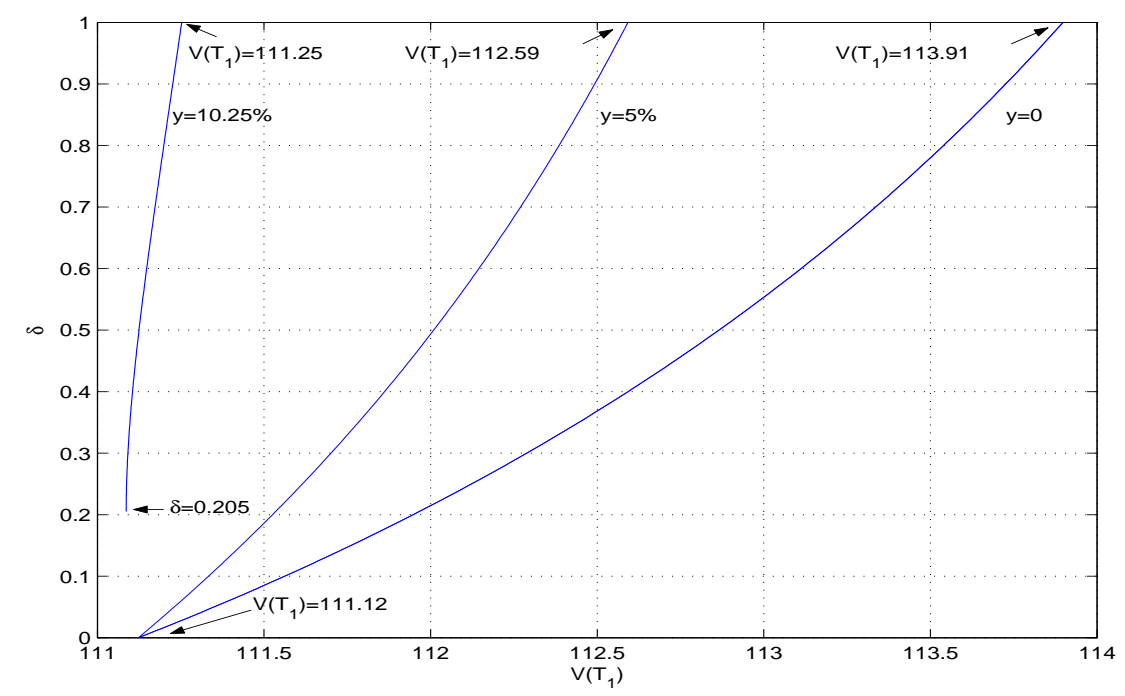

Figure 3: The graph plots the competitive equilibrium exercise policy $\delta$ when the firm pays an extraordinary dividend that is equal to $y \%$ of the cash proceeds from exercise. There are $\alpha$ units of an upstream warrant $\bar{W}$ of strike $X$ and expiration date $T_{2}$. The parameters used to compute the exercise policy are: $\alpha=1, \bar{\alpha}=1, r=0, X=90, \bar{X}=100, T_{2}-T_{1}=5$.

firm pays an extraordinary dividend per share of $D$ equal to a fraction of the exercise proceeds, i.e., for a given $y, 0 \leq y \leq 1$,

$$
D=y\left(\frac{\sum_{k=1}^{K} \delta^{k} \alpha^{k} X}{1+\sum_{k=1}^{K} \delta^{k} \alpha^{k}}\right) .
$$

At $T_{1}, \bar{W}$ is priced at the ex-dividend value, $V^{+}\left(T_{1}\right)=\left(V\left(T_{1}\right)+\sum_{k=1}^{K} \delta^{k} \alpha^{k} X-D\right) /(1+$ $\left.\sum_{k=1}^{K} \delta^{k} \alpha^{k}\right)$. The extraordinary dividend, if sufficiently high, ensures that $\partial \bar{W} / \partial \delta<0$ for all firm values where the warrant is at- or in-the-money. Part B of the Proposition and Lemma 2 then ensure a block exercise policy. ${ }^{10}$

Below, we provide illustrations of the competitive equilibrium policy for different levels of $y$. Figure 3 plots some of these equilibrium policies.

${ }^{10}$ Differentiating $\bar{W}$ with respect to $\delta^{k}$ and simplifying using (1), we get (see Appendix B),

$$
\begin{aligned}
\frac{\partial \bar{W}}{\partial \delta^{k}} & =\frac{\bar{\alpha} \alpha^{k}}{\left(1+\bar{\alpha}^{+}\right)\left(1+\sum_{k^{\prime}=1}^{K} \delta^{k^{\prime}} \alpha^{k^{\prime}}\right)^{2}}[\bar{W}-\Delta(V-(1-y) X)] \\
& \left.=\frac{\bar{\alpha}^{+} \alpha^{k}}{\left(1+\bar{\alpha}^{+}\right)\left(1+\sum_{k^{\prime}=1}^{K} \delta^{k^{\prime}} \alpha^{k^{\prime}}\right)}\left[(1-\Delta) \bar{W}-\Delta\left(1+\sum_{k^{\prime}=1}^{K} \delta^{k^{\prime}} \alpha^{k^{\prime}}\right)(S-X)-y \Delta X\right)\right],
\end{aligned}
$$

where, as earlier defined, $\Delta$ is the "delta" of the call. Observe that for sufficiently high $y$ and $S=X, \partial \bar{W} / \partial \delta^{k}<$ 0 . Moreover, if $\partial \bar{W} / \partial \delta^{k}$ is negative for $S=X$, then it is also negative for $S>X$. 
- Example 1: $y=5 \%$.

The competitive equilibrium exercise policy is,

$$
\begin{aligned}
\delta & =1 \quad \text { if } V\left(T_{1}\right)>112.59 \\
& =\hat{\delta}\left(V\left(T_{1}\right)\right) \quad \text { if } 111.12 \leq V\left(T_{1}\right) \leq 112.59 \\
& =0 \quad \text { if } V\left(T_{1}\right)<111.12
\end{aligned}
$$

where $0 \leq \hat{\delta}\left(V\left(T_{1}\right)\right) \leq 1$. As the dividend amount is small, $\partial \bar{W} / \partial \delta>0$ for $V \in \mathbf{V}$ and $\delta \in[0,1]$, and there is a partial exercise equilibrium. In comparison with the equilibrium policy for $y=0$, more warrants are partially exercised as the dividend reduces the transfer of wealth from warrant- and shareholders to the individuals who hold $\bar{W}$.

- Example 2: $y=10.25 \%$.

The competitive equilibrium exercise policy is,

$$
\begin{aligned}
\delta & =1 \quad \text { if } V\left(T_{1}\right)>111.25 \\
& =\hat{\delta}\left(V\left(T_{1}\right)\right) \quad \text { if } 111.086 \leq V\left(T_{1}\right) \leq 111.25 \\
& =0 \quad \text { if } V\left(T_{1}\right) \leq 111.086
\end{aligned}
$$

where $0.205 \leq \hat{\delta}\left(V\left(T_{1}\right) \leq 1\right.$. For firm values corresponding to $\mathbf{V}, \partial \bar{W} / \partial \delta$ is not strictly positive for $\delta<0.205$ (in particular, at $V\left(T_{1}\right)=111.086, \partial \bar{W} / \partial \delta<0$ for $\delta<0.205$ ). Although the warrant may be partially exercised in equilibrium, there is no equilibrium policy where $\delta$ is in $(0,0.205)$. At $V\left(T_{1}\right)=111.086$, there are two equilibria corresponding to $\delta=0$ and $\delta=0.205$, i.e., the equilibrium policy is no longer convex-valued.

- Example 3: $y=30 \%$ and $y=100 \%$.

For $y=30 \%$, the block exercise policy is,

$$
\begin{aligned}
\delta & =0, & & V\left(T_{1}\right) \leq 106.52 \\
& =1, & & V\left(T_{1}\right) \geq 106.52,
\end{aligned}
$$

with no equilibrium policy where $\delta \in(0,1)$. At the firm value of 106.52 , there are two equilibria corresponding to $\delta=0$ and $\delta=1$ and stock prices of $\$ 87.02$ and $\$ 90$, respectively. The corresponding values of $\bar{W}$ are $\$ 19.50$ and $\$ 16.52$, respectively. The equilibrium policy is also block, of course, for every $y$ greater than $30 \%$. For $y=100 \%$, 
the block exercise policy is,

$$
\begin{aligned}
\delta & =0, & V\left(T_{1}\right) \leq 94.31 \\
& =1, & V\left(T_{1}\right) \geq 94.31
\end{aligned}
$$

with corresponding stock prices of $\$ 78.93$ and $\$ 90$, respectively. The $14 \%$ jump in the stock price at the exercise threshold is greater than that for $y=30 \%$ as the magnitude of wealth transfer to the stock-holders is larger.

The above illustrations also demonstrate that the equilibrium exercise policy depends on how exercise proceeds are used by the firm. Under what circumstances should we expect to observe the block or the partial equilibrium strategy? When shareholders of a firm can prevent wealth transfer away from themselves to the holders of $\bar{W}$, we will not observe a partial equilibrium strategy. Shareholders can most effectively accomplish this by not investing the exercise proceeds. ${ }^{11}$ This suggests that the partial exercise strategy is intimately linked to the decision of the firm to have warrants and similar securities as a source of capital in the capital structure for investment purposes. Green (1984), Stein (1992) and Mayers (1998), amongst others, provide reasons why firms may choose warrants and convertible bonds as a source of capital. When such a reason does not exist, firms may institute policies for preventing wealth transfer as, for instance, using $100 \%$ of the proceeds of exercise to pay an extraordinary dividend. The significance of the use of exercise proceeds in determining equilibrium prices motivates further empirical work in understanding how exactly firms use exercise proceeds. ${ }^{12}$

\subsection{The Competitive vs. the Monopolist Equilibrium}

We can relate the competitive partial equilibrium policy to the monopolist's policy. When comparing equilibrium policies in Figure 2, we observed that fewer warrants are partially exercised by the monopolist. We make this statement precise. As the number of warrants exercised is related to the magnitude of wealth transfer to $\bar{W}$, we can relate the competitive and monopolist's exercise policy through $\bar{W}$. Let the fraction of warrants partially exercised

\footnotetext{
${ }^{11}$ In addition, when $\bar{W}$ is another warrant, then the firm can also limit or prevent wealth transfer to the holder of $\bar{W}$ by investing the proceeds into projects of lower riskiness. However, this strategy is not effective if, instead, $\bar{W}$ is risky debt.

${ }^{12}$ Mayers (1998) finds that capital expenditure increases after forced conversion of convertible bonds and suggests that these securities are used to fund growth opportunities. To investigate how many firms pay extraordinary dividends, we considered a sample of 675 firms that issued convertible bonds over the period 1981-2004. Of these, only 5 firms paid extraordinary dividends after the year of issuance (481 or $71 \%$ of these firms did not pay ordinary nor extraordinary dividends after issuance). Spatt and Sterbenz (1988) in their footnote 7 note that in their sample the majority of firms that issue warrants do not pay dividends.
} 
in equilibrium in the non-competitive equilibrium at $V\left(T_{1}\right)=V$ be denoted as $\delta^{m}$. From equation (6), $S\left(V, \delta^{m}\right)=X-\delta^{m} \partial S\left(V, \delta^{m}\right) / \partial \delta$. Substituting this value of $S$ in (1), we get,

$$
V=X-\left(1+\delta^{m} \alpha\right) \delta^{m} \frac{\partial S\left(V, \delta^{m}\right)}{\partial \delta}+\bar{W}\left(V, \delta^{m}\right)
$$

At this same firm value, let $\delta^{c}$ be the number exercised in the competitive equilibrium. From Part A of Proposition $1, S\left(V, \delta^{c}\right)=X_{1}$, and,

$$
V=X_{1}+\bar{W}\left(V, \delta^{c}\right)
$$

Subtracting (17) from (18), we get,

$$
\begin{aligned}
\bar{W}\left(V, \delta^{c}\right)-\bar{W}\left(V, \delta^{m}\right) & =-\left(1+\delta^{m} \alpha_{1}\right) \delta^{m} \frac{\partial S\left(V, \delta^{m}\right)}{\partial \delta} \\
& =\left(1+\delta^{m} \alpha\right) \delta^{m} \frac{\partial \bar{W}\left(V, \delta^{m}\right)}{\partial \delta}
\end{aligned}
$$

where the last equality uses equation (5) to substitute for $\partial S / \partial \delta$. As $\bar{W}$ is strictly increasing in $\delta$, it follows that $\delta^{m}<\delta^{c}{ }^{13}$ As we know how to compute $\delta^{c}$ and $\bar{W}\left(V, \delta^{c}\right)$, we can use equation (19) to compute $\delta^{m}$, and (upper) bound the warrant price.

\section{Valuation and Implementation}

We observed that the equilibrium exercise policy depends on the securities in the capital structure, and, in turn, the value of these securities will depend on the equilibrium exercise policy. In deriving the equilibrium policy, we need to consistently value all securities, including warrants, in the capital structure. We provide guidance on how this can be done given that we observe the stock price $S(t)$ but not $V(t)$.

For generality, we allow the warrants expiring at time $T_{1}$ to have $J \geq 1$ distinct strikes and dilution factors. Specifically, let there be $\alpha_{j}$ of each warrant $W_{j}$ of strike $X_{j}, j=1, \ldots, J$, $X_{1}<X_{2}<\ldots<X_{J}<\infty$. Correspondingly, we write equation (1) as,

$$
V(t)=S(t)+\sum_{j=1}^{J} \alpha_{j} W_{j}(t)+\bar{W}(t),
$$

\footnotetext{
${ }^{13}$ One way of observing this is to apply the Mean Value Theorem to the LHS of (19), which gives $\delta^{m}=$ $\delta^{c}-\left(1+\delta^{m} \alpha\right) \delta^{m}\left(\frac{\partial \bar{W}\left(V, \delta^{m}\right)}{\partial \delta^{m}} / \frac{\partial \bar{W}(V, \tilde{\delta})}{\partial \tilde{\delta}}\right)$ for $\delta^{m}<\tilde{\delta}<\delta^{c}$.
} 
Each of these warrants may be held by $K_{j} \geq 1$ individuals. The exercise policy of the $k$ 'th individual holding a portion of $W_{j}$ is denoted as $\delta_{j}^{k}$.

\subsection{Valuation when $\bar{W} \equiv 0$}

If there are no other securities in the capital structure besides the stock and $J$ warrants, the equilibrium exercise policy is a block equilibrium. This, in turn, determines the payoff of the warrant for each $V\left(T_{1}\right)$. The exercise threshold and payoff of the warrant are given as follows. For each of $j$ warrants, $j=1, . ., J$ :

1. Exercise Threshold: The warrant of strike $X_{j}$ is fully exercised if $V\left(T_{1}\right) \geq \bar{V}_{j}$, where $\bar{V}_{j}=X_{j}+\sum_{j^{\prime}=1}^{j} \alpha_{j^{\prime}}\left(X_{j}-X_{j^{\prime}}\right)$, and expires worthless otherwise.

2. Payoff: The payoff of warrant of strike $X_{j}$ is:

$$
\begin{aligned}
W_{j}\left(T_{1}\right) & =0, \quad V\left(T_{1}\right)<\bar{V}_{j} \\
& =\frac{1}{1+\bar{\alpha}_{j+l}}\left(V\left(T_{1}\right)-X_{j}^{j+l}\right), \quad \bar{V}_{j+l} \leq V\left(T_{1}\right)<\bar{V}_{j+l+1}
\end{aligned}
$$

for $l=0,1, . ., J-j, X_{j}^{j+l} \equiv X_{j}+\sum_{j^{\prime}=1}^{j+l} \alpha_{j^{\prime}}\left(X_{j}-X_{j^{\prime}}\right)$, and $\bar{\alpha}_{j} \equiv \sum_{j^{\prime}=1}^{j} \alpha_{j^{\prime}}$.

The exercise threshold can be derived by noting that Lemma 1 also applies when $J>1$, i.e., a warrant is fully exercised if it is in-the-money. From (20), $S\left(T_{1}\right) \gtreqless X_{j}$ is equivalent to $V\left(T_{1}\right) \gtreqless \bar{V}_{j}$, where $\bar{V}=X_{j}+\sum_{j^{\prime}=1}^{j} \alpha_{j^{\prime}}\left(X_{j}-X_{j^{\prime}}\right)$. The payoff of the warrant in equation (21) follows by substituting the number of exercised warrants into equation (20) and re-arranging to express the payoff in terms of $V\left(T_{1}\right)$. For any $V\left(T_{1}\right)$, the payoff of the warrant depends on the total number of exercised warrants. As the number of in-the-money warrants depends on the level of $V\left(T_{1}\right)$, the slope of the payoff of the warrant changes as $V\left(T_{1}\right)$ increases, and more warrants are in-the-money. Over the range $0<V\left(T_{1}\right)<\infty$, the payoff for the $j$ 'th warrant must be described separately for each of $J-j+1$ segments. Despite the complicated payoff structure of the warrant, we can derive a simple recursive formula that generalizes the single European warrant pricing formula of Galai and Schneller (1978).

Proposition 2 Suppose the capital structure of the firm consists of the stock and $J \geq 1$ warrants of maturity $T_{1}$ and strikes $X_{j}, j=1,2, \ldots, J$. Then the value of these $J$ warrants is given as follows. For $j<J$,

$$
W_{j}(t)=\frac{1}{1+\bar{\alpha}_{j}}\left(C\left(V(t), \bar{V}_{j} ; T_{1}-t\right)-C\left(V(t), \bar{V}_{j+1} ; T_{1}-t\right)\right)+W_{j+1}(t),
$$


and, for $j=J$,

$$
W_{J}(t)=\frac{1}{1+\bar{\alpha}_{J}} C\left(V(t), \bar{V}_{J} ; T_{1}-t\right)
$$

where $\bar{V}_{j}$ is defined as

$$
\bar{V}_{j} \equiv X_{j}^{j}=X_{j}+\sum_{j^{\prime}=1}^{j} \alpha_{j^{\prime}}\left(X_{j}-X_{j^{\prime}}\right)
$$

and $\bar{\alpha}_{j} \equiv \sum_{j^{\prime}=1}^{j} \alpha_{j^{\prime}}, C(V(t), X ; T-t)=E_{t}^{Q} e^{-r(T-t)} \max \left(V\left(T_{1}\right)-X, 0\right)$.

Proof of Proposition 2: See Appendix.

The proof of the proposition uses the fact noted earlier that when all warrants have the same maturity, the payoff of a warrant is piecewise linear in $V\left(T_{1}\right)$ with the number of segments equal to the number of distinct warrants that are exercised. Because of this piecewise linearity, a warrant bull spread is equivalent to the corresponding (diluted) spread in call options. Recall that when there is a single warrant series, a warrant is equivalent to a diluted call on the value of the firm. The expression for a single warrant is a special case when one of the options in the option spread has a strike of infinity (and a price of 0 ), so that the existing single warrant pricing theory of equation (7) is subsumed in Proposition $2 .{ }^{14}$

\subsection{Valuation with $\bar{W} \neq 0$}

When the capital structure of the firm has other securities and $\partial \bar{W} \neq 0$, we first need to compute the equilibrium exercise policy. We assume below that the securities are valued under a competitive equilibrium. ${ }^{15}$

Given $\delta_{j}\left(V\left(T_{1}\right)\right)$, the payoff of each exercised warrant, $W_{j}=\left(S\left(T_{1}\right)-X\right)^{+}$, may be written in terms of $V\left(T_{1}\right)$ using equation (20). The payoff of $W_{j}$ is,

$$
W_{j}\left(T_{1}\right)=\frac{1}{1+\bar{\alpha}_{J}}\left(V\left(T_{1}\right)-\hat{X}_{j}\left(V\left(T_{1}\right)\right)\right)
$$

\footnotetext{
${ }^{14}$ If we substitute out the recursive structure of Proposition 2, we get,

$$
W_{j}(t)=\frac{C\left(V(t), \bar{V}_{j} ; T_{1}-t\right)}{1+\bar{\alpha}_{j}}-\sum_{j^{\prime}=j+1}^{J} \frac{\alpha_{j^{\prime}} C\left(V(t), \bar{V}_{j^{\prime}} ; T_{1}-t\right)}{\left(1+\bar{\alpha}_{j^{\prime}}\right)\left(1+\bar{\alpha}_{j^{\prime}-1}\right)}
$$

A similar expression has also been independently noted by Darsinos and Satchell (2002).

${ }^{15}$ Proposition 1 can be generalized to allow for $J>1$ by applying it to each warrant of strike $X_{j}$.
} 
where

$$
\hat{X}_{j}\left(V\left(T_{1}\right)\right) \equiv X_{j}+\sum_{j^{\prime}=1}^{J} \delta_{j^{\prime}}\left(V\left(T_{1}\right)\right) \alpha_{j^{\prime}}\left(X_{j}-X_{j^{\prime}}\right)+\bar{W}\left(T_{1}\right),
$$

and $\bar{\alpha}_{J} \equiv \sum_{j^{\prime}=1}^{J} \delta_{j^{\prime}} \alpha_{j^{\prime}}$.

Equations (22) and (23) express the payoff of each warrant in terms of a state-dependent adjusted strike, $\hat{X}_{j}\left(V\left(T_{1}\right)\right)$, that depends on the value of the firm at exercise. $\hat{X}_{j}$ accounts for the effect of both exercised warrants and downstream securities on the payoff. Unlike the case when $\bar{W}=0$, the payoff is non-linear, and therefore, there does not exist any equivalence between warrant and option prices as in Proposition 2. Non-linearity arises from the dependence of the payoff on the value of upstream securities, which in turn depends on how many warrants are exercised at $T_{1}$.

Given the exercise policy, the value of each warrant is computed numerically using equations (22) and (23) by taking expectations of the discounted payoff. Given the value of the warrants and other securities, we can compute the exercise policy. Thus, when only $S(t)$ is observable, an iterative procedure will have to be implemented to ensure consistency of both exercise strategies and prices of the securities.

For illustration, consider once again our two-warrant example. Conditioned on an exercise policy of $\hat{\delta}\left(V\left(T_{1}\right)\right)$, the payoff of $W$ at $T_{1}$ is (from equation 22),

$$
W\left(T_{1}\right)=\frac{1}{1+\hat{\delta} \alpha} \operatorname{Max}\left(V\left(T_{1}\right)-\hat{X}, 0\right)
$$

where $\hat{X}=X+\bar{W}\left(T_{1}\right)$. The value of $\bar{W}$ at $T_{1}$ is,

$$
\bar{W}\left(T_{1}\right)=\frac{\bar{\alpha}}{1+\bar{\alpha}^{+}} C\left(V^{+}\left(T_{1}\right), \bar{X} ; T_{2}-T_{1}\right),
$$

where $V^{+}=\left(V\left(T_{1}\right)+\hat{\delta} X\right) /(1+\hat{\delta} \alpha)$, and $\bar{\alpha}^{+}=\bar{\alpha} /(1+\hat{\delta} \alpha)$. Given these values of $W\left(T_{1}\right)$ and $\bar{W}\left(T_{1}\right)$, the prices of these warrants at $t<T_{1}$ is the solution to the set of equations,

$$
\begin{aligned}
V(t) & =S(t)+\alpha W(t)+\bar{W}(t), \\
W(t) & =\mathrm{E}_{t}^{Q} e^{-r\left(T_{1}-t\right)} W\left(T_{1}\right), \\
\bar{W}(t) & =\mathrm{E}_{t}^{Q} e^{-r\left(T_{1}-t\right)} \bar{W}\left(T_{1}\right) .
\end{aligned}
$$

The expectation can be computed by usual numerical methods as, for example, using a binomial tree. We provide below an example of an iterative algorithm that can be used for valuation. 
1. Start with an initial guess of $V(t)$ (say, $V(t)=S(t)$ ).

2. Determine the exercise policy, $\hat{\delta}\left(V\left(T_{1}\right)\right)$. If there is a partial exercise equilibrium, solve equation (15), $V(\hat{\delta})=X+\bar{W}(V(\hat{\delta}), \hat{\delta})$ for every $\hat{\delta} \in[0,1]$. Store the vector, $\hat{\delta}\left(V\left(T_{1}\right)\right)$.

3. Given $V(t)$, construct the grid of $V\left(T_{1}\right)$. Then, for each $V\left(T_{1}\right)$ and corresponding $\hat{\delta}\left(V\left(T_{1}\right)\right)$, calculate the value of all securities at $T_{1}$, i.e., compute the value of $W\left(T_{1}\right)$ and $\bar{W}\left(T_{1}\right)$ using equations (24) and (25), respectively.

4. Calculate the values of $W(t)$ and $\bar{W}(t)$ by taking expectations of the discounted payoff under $Q$,

$$
\begin{aligned}
& W(t)=\mathrm{E}_{t}^{Q} e^{-r\left(T_{1}-t\right)} W\left(T_{1}\right) \\
& \bar{W}(t)=\mathrm{E}_{t}^{Q} e^{-r\left(T_{1}-t\right)} \bar{W}\left(T_{1}\right),
\end{aligned}
$$

5. Estimate the new value of $V(t)=S(t)+\alpha W(t)+\bar{W}(t)$, and iterate to convergence by repeating steps $2-4$.

\section{Conclusion}

Exercise of a warrant potentially impacts all risky securities in the capital structure, besides the stock. Depending on how the firm uses the exercise proceeds, exercise may result in wealth transfer to or away from the holders of these other securities. When the sum total of these securities increase in value, a warrant-holder adjusts the quantity exercised to mitigate the adverse impact on the value of his warrants. However, the extent to which the warrant-holder is successful in doing so is affected by the concentration of warrant-holding. In the extreme case, when warrants are competitively held by a large number of warrant-holders, the net payoff to exercise is zero for a range of firm values. When the sum total declines in value, the warrant-holders exercise as a block. That is, if they choose to exercise, they exercise all their warrants. Thus, exercise results in a jump in the stock price with a corresponding decline in the value of the other securities.

In summary, because all risky securities are potentially impacted by warrant exercise, the warrant-holder's equilibrium exercise policy depends on the entire the capital structure of the firm. Warrant pricing theory that does not explicitly account for the entire capital structure of the firm is simply incomplete. For instance, in existing European warrant theory where there is only one warrant and stock in the capital structure, the concentration of warrant-holding 
does not affect warrant exercise or price. On the contrary, we demonstrate that it may play an important role in determining both.

Our observation of the importance of determining equilibrium policies in the context of the entire capital structure of the firm applies more generally. For instance, almost two decades after Constantinides (1984), the exercise policy and valuation of the American warrant is well understood only when the capital structure has only stock and warrants. The theory developed here for the European warrant motivates the corresponding development of a more general theory for the American warrant. 


\section{Appendix A}

\section{Proof of Proposition 1, Part A:}

Consider $V \in \mathbf{V}$, i.e., the set of firm values at $T_{1}$ defined by substituting $S\left(T_{1}\right)=X$ in equation (1),

$$
V=X+\bar{W}(V, \delta)
$$

where $\delta \in[0,1]$.

Write (26) as

$$
V(\hat{\delta})=X+\bar{W}(V(\hat{\delta}), \hat{\delta})
$$

which is the inverse problem of determining $V$ for a given $\delta=\hat{\delta}$, and is a fixed point problem. Uniqueness of the fixed point follows as $\partial \bar{W} / \partial V$ is strictly less than 1 . Define firm values for which the corresponding value of $\hat{\delta}$ is 0 and 1 as $V^{d} \equiv V(0)$ and $V^{u} \equiv V(1)$, respectively. Continuity of $V$ in $\hat{\delta}$ follows from the continuity of $\bar{W}(V, \hat{\delta})$. Equating the $\hat{\delta}$ derivative of both

sides of (27), and noting that $\left.\frac{\partial \bar{W}}{\partial \hat{\delta}}\right|_{V}>0$ and that $\frac{\partial \bar{W}}{\partial V}<1$, a rearrangement to compute $V^{\prime}(\hat{\delta})$ shows it is always positive. By the Inverse Function Theorem, it follows that $\hat{\delta}(V)$ exists, and that it is increasing. Thus, $\hat{\delta}(V)$ defined by $S=X$ is an exercise policy establishing the main claim under part iii. Moreover, the uniqueness of $\hat{\delta}(V)$ allows us to identify $\hat{\delta}\left(V^{d}\right)=0$ and $\hat{\delta}\left(V^{u}\right)=1$. which proves A and B under iii. Parts C and D are addressed below.

Consider what happens when some exercise policy, $\delta$, other than that specified by $\hat{\delta}(V)$, is used. In this case, equation (1) becomes

$$
V=S(V, \delta)+\delta \alpha(S(V, \delta)-X)+\bar{W}(V, \delta)
$$

Fixing $V$, subtract equation (27) from (28) to obtain

$$
(1+\delta \alpha)(S(V, \delta)-X)=\bar{W}(V, \hat{\delta})-\bar{W}(V, \delta)
$$

Items C and D of part iii follow from (29) and the assumption that $\bar{W}$ is an increasing function of $\delta$.

To complete the proof of the claims under parts i and ii, subtract the defining equations for $V^{d}$ and $V^{u}$ from equation (28), for some $V$ with the exercise policy $\delta$. To prove part i (part 
ii is similar), We have

$$
\begin{aligned}
V-V^{d}-(1+\delta \alpha)(S(V, \delta)-X) & =\bar{W}(V, \delta)-\bar{W}\left(V^{d}, 0\right) \\
& =\bar{W}(V, \delta)-\bar{W}\left(V^{d}, \delta\right)+\bar{W}\left(V^{d}, \delta\right)-\bar{W}\left(V^{d}, 0\right) \\
& =\frac{\partial \bar{W}\left(V^{d}, \tilde{\delta}\right)}{\partial \delta} \delta+\frac{\partial \bar{W}(\tilde{V}, \delta)}{\partial V}\left(V-V^{d}\right)
\end{aligned}
$$

where the latter step involving $\tilde{V}$ and $\tilde{\delta}$ uses the Mean Value Theorem. As $0<\partial \bar{W} / \partial V<1$ and $\bar{W}$ increases with $\delta$, equation (32) implies $S<X$.

\section{Proof of Proposition 1, Part B:}

First, as $\partial \bar{W} / \partial V<1, \bar{V}$ is uniquely defined by substituting $S\left(T_{1}\right)=X$ and $\delta=1$ in equation $(1)$,

$$
\bar{V}=X+\bar{W}(\bar{V}, 1)
$$

To prove part [ii.], consider an exercise policy $\delta \in[0,1)$ and $V\left(T_{1}\right)=\bar{V}$. Substituting in equation (1), we get,

$$
\bar{V}=S(\bar{V}, \delta)+\delta \alpha(S(\bar{V}, \delta)-X)+\bar{W}(\bar{V}, \delta) .
$$

Now substract equation (33) from (34) to give,

$$
\begin{aligned}
-(1+\delta \alpha)(S(\bar{V}, \delta)-X) & =\bar{W}(\bar{V}, \delta)-\bar{W}(\bar{V}, 1) \\
& =\frac{\partial \bar{W}(\bar{V}, \tilde{\delta})}{\partial \delta}(\delta-1)
\end{aligned}
$$

where the latter step uses the Mean Value Theorem for $\tilde{\delta} \in(\delta, 1)$. As $\partial \bar{W} / \partial \delta<0$, and $\delta<1$, it follows that $S(\bar{V}, \delta)<X$. This proves part [iii.].

To prove parts [i.] and [iii.] consider any other firm value $V \neq \bar{V}$ with an exercise policy $\delta \in[0,1]$. Substituting in equation (1), we get,

$$
V=S(V, \delta)+\delta \alpha(S(V, \delta)-X)+\bar{W}(V, \delta)
$$

Substituting the identical exercise policy and firm value $\bar{V}$ in equation (1) results in,

$$
\bar{V}=S(\bar{V}, \delta)+\delta \alpha(S(\bar{V}, \delta)-X)+\bar{W}(\bar{V}, \delta) .
$$


Subtract (38) from (37) and apply the Mean Value Theorem to give,

$$
\begin{aligned}
V-\bar{V} & =(1+\delta \alpha)(S(V, \delta)-S(\bar{V}, \delta))+\bar{W}(V, \delta)-\bar{W}(\bar{V}, \delta) \\
& =(1+\delta \alpha)(S(V, \delta)-S(\bar{V}, \delta))+\frac{\partial \bar{W}(\tilde{V}, \delta)}{\partial V}(V-\bar{V})
\end{aligned}
$$

As $0<\partial W / \partial V<1$, it follows that $V<\bar{V}$ implies that $S(V, \delta)<S(\bar{V}, \delta)$, and $V>\bar{V}$ implies that $S(V, \delta)>S(\bar{V}, \delta)$. From part [ii.] and the definition of $\bar{V}$, we know $S(\bar{V}, \delta)$ reaches its supremum at $S(\bar{V}, 1)=X$. Therefore, $V<\bar{V}$ implies that $S(V, \delta)<S(\bar{V}, \delta) \leq X$, and $V>\bar{V}$ implies $S(V, 1)>S(\bar{V}, 1)=X$.

\section{Proof of Proposition 2:}

In the following proof, we adopt the notation $C_{j} \equiv C\left(V(t), \bar{V}_{j} ; T_{1}-t\right)$, and, without loss of generality, we assume the risk-free rate, $r$, to be equal to zero. Also, define $\bar{\alpha}_{J} \equiv \sum_{j=1}^{J} \alpha_{j}$.

First, note that $C_{j}=\int_{\bar{V}_{j}}^{\infty}\left(V\left(T_{1}\right)-\bar{V}_{j}\right) d Q$. Consider the $J$ 'th warrant, $W_{J}$. Given the payoff $W_{J}$, it immediately follows that $W_{J}=C_{J} /\left(1+\bar{\alpha}_{J}\right)$. Now consider the $j$ 'th warrant, $j<J$. From (21), the value of $W_{j}$ under the risk-neutral distribution, $Q$, is,

$$
W_{j}(t)=\int_{X_{j}^{j}}^{X_{j+1}^{j+1}} \frac{V\left(T_{1}\right)-X_{j}^{j}}{1+\bar{\alpha}_{j}} d Q+\int_{X_{j+1}^{j+1}}^{X_{j+2}^{j+2}} \frac{V\left(T_{1}\right)-X_{j}^{j+1}}{1+\bar{\alpha}_{j+1}} d Q+\ldots+\int_{X_{J}^{J}}^{\infty} \frac{V\left(T_{1}\right)-X_{j}^{J}}{1+\bar{\alpha}_{J}} d Q
$$

Therefore,

$W_{j}(t)-W_{j+1}(t)=\int_{X_{j}^{j}}^{X_{j+1}^{j+1}} \frac{V\left(T_{1}\right)-X_{j}^{j}}{1+\bar{\alpha}_{j}} d Q+\int_{X_{j+1}^{j+1}}^{X_{j+2}^{j+2}} \frac{X_{j+1}^{j+1}-X_{j}^{j+1}}{1+\bar{\alpha}_{j+1}} d Q+\ldots+\int_{X_{J}}^{\infty} \frac{X_{j+1}^{J}-X_{j}^{J}}{1+\bar{\alpha}_{J}} d Q$.

Consider the first term in equation (42),

$$
\begin{aligned}
\int_{X_{j}^{j}}^{X_{j+1}^{j+1}} \frac{V\left(T_{1}\right)-X_{j}^{j}}{1+\bar{\alpha}_{j}} d Q & =\frac{1}{1+\bar{\alpha}_{j}} C_{j}-\int_{X_{j+1}^{j+1}}^{\infty} \frac{V\left(T_{1}\right)-X_{j}^{j}}{1+\bar{\alpha}_{j}} d Q \\
& =\frac{1}{1+\bar{\alpha}_{j}} C_{j}-\frac{1}{1+\bar{\alpha}_{j}} C_{j+1}-\int_{X_{j+1}^{j+1}}^{\infty}\left(X_{j+1}-X_{j}\right) d Q
\end{aligned}
$$

where the last equality uses the definition of $C_{j+1}$, and substitutes $X_{j}^{j+1}$ for $X_{j}^{j}$, using the identity,

$$
X_{j+1}^{j+1}-X_{j}^{j}=\left(1+\bar{\alpha}_{j}\right)\left(X_{j+1}-X_{j}\right)
$$

Finally, the sum of the second to the last terms of equation (42) may be simplified by using 
the identity,

$$
X_{j+1}^{k}-X_{j}^{k}=\left(1+\bar{\alpha}_{k}\right)\left(X_{j+1}-X_{j}\right)
$$

to give

$$
\int_{X_{j+1}^{j+1}}^{X_{j+2}^{j+2}} \frac{X_{j+1}^{j+1}-X_{j}^{j+1}}{1+\bar{\alpha}_{j+1}} d Q+\ldots+\int_{X_{J}}^{\infty} \frac{X_{j+1}^{J}-X_{j}^{J}}{1+\bar{\alpha}_{J}} d Q=\int_{X_{j+1}^{j+1}}^{\infty}\left(X_{j+1}-X_{j}\right) d Q
$$

It follows from equations (44) and (47) that

$$
W_{j}(t)=\frac{1}{1+\bar{\alpha}_{j}}\left(C_{j}-C_{j+1}\right)+W_{j+1}(t) .
$$

Note that the statement of the Proposition uses the notation $\bar{V}_{j} \equiv X_{j}^{j}$. 


\section{Appendix B}

This appendix provides derivations for the formulae for $\partial \bar{W} / \partial \delta^{k}$ used in footnote 10, equation (12), and footnote 8 . To compute $\partial \bar{W} / \partial \delta^{k}$, recall, from (9) that $\bar{W}=\bar{\alpha} \frac{C\left(V^{+}\right)}{1+\bar{\alpha}^{+}}$and $\bar{\alpha}^{+}=$ $\frac{\bar{\alpha}}{1+\sum_{k^{\prime}}^{K} \delta^{k^{\prime}} \alpha^{k^{\prime}}}$, and from equation (4) that

$$
V^{+}=\frac{V+(1-y) \sum_{k^{\prime}=1}^{K} \delta^{k^{\prime}} \alpha^{k^{\prime}} X}{1+\sum_{k^{\prime}=1}^{K} \delta^{k^{\prime}} \alpha^{k^{\prime}}}
$$

Differentiating $\bar{\alpha}^{+}$and $V^{+}$with respect to $\delta^{k}$, we get,

$$
\frac{\partial \bar{\alpha}^{+}}{\partial \delta^{k}}=\frac{-\bar{\alpha} \alpha^{k}}{\left(1+\sum_{k^{\prime}=1}^{K} \delta^{k^{\prime}} \alpha^{k^{\prime}}\right)^{2}},
$$

and

$$
\begin{aligned}
\frac{\partial V^{+}}{\partial \delta^{k}} & =\frac{\left(1+\sum_{k^{\prime}=1}^{K} \delta^{k^{\prime}} \alpha^{k^{\prime}}\right)(1-y)\left(\alpha^{k} X\right)-\left(\alpha^{k}\right)\left(V+(1-y) \sum_{k^{\prime}=1}^{K} \delta^{k^{\prime}} \alpha^{k^{\prime}} X\right)}{\left(1+\sum_{k^{\prime}=1}^{K} \delta^{k^{\prime}} \alpha^{k^{\prime}}\right)^{2}} \\
& =\frac{-\alpha^{k}(V-(1-y) X)}{\left(1+\sum_{k^{\prime}=1}^{K} \delta^{k^{\prime}} \alpha^{k^{\prime}}\right)^{2}}
\end{aligned}
$$

We can now compute $\partial \bar{W} / \partial \delta^{k}$ by differentiating (9) (where we define $\Delta \equiv \partial C\left(V^{+}\right) / \partial V^{+}$),

$$
\begin{aligned}
\frac{\partial \bar{W}}{\partial \delta^{k}} & =\frac{-\bar{\alpha}}{\left(1+\bar{\alpha}^{+}\right)^{2}} \frac{\partial \bar{\alpha}^{+}}{\partial \delta^{k}} C\left(V^{+}\right)+\frac{\bar{\alpha}}{1+\bar{\alpha}^{+}} \Delta \frac{\partial V^{+}}{\partial \delta^{k}} \\
& =\frac{\bar{W}}{\left(1+\bar{\alpha}^{+}\right)} \frac{-\partial \bar{\alpha}^{+}}{\partial \delta^{k}}+\frac{\bar{\alpha}}{\left(1+\bar{\alpha}^{+}\right)} \Delta \frac{\partial V^{+}}{\partial \delta^{k}} \\
& =\frac{\bar{W}}{\left(1+\bar{\alpha}^{+}\right)} \frac{\bar{\alpha} \alpha^{k}}{\left(1+\sum_{k^{\prime}=1}^{K} \delta^{k^{\prime}} \alpha^{k^{\prime}}\right)^{2}}+\frac{\bar{\alpha}}{\left(1+\bar{\alpha}^{+}\right)} \Delta \frac{-\alpha^{k}(V-(1-y) X)}{\left(1+\sum_{k^{\prime}=1}^{K} \delta^{k^{\prime}} \alpha^{k^{\prime}}\right)^{2}}, \\
& =\frac{\bar{\alpha}^{+} \alpha^{k}}{\left(1+\bar{\alpha}^{+}\right)\left(1+\sum_{k^{\prime}=1}^{K} \delta^{k^{\prime}} \alpha^{k^{\prime}}\right)}[\bar{W}-\Delta(V-(1-y) X)] .
\end{aligned}
$$

Now noting from equation (1) that $V-(1-y) X=\left(1+\sum_{k^{\prime}=1}^{K} \delta^{k^{\prime}} \alpha^{k^{\prime}}\right)(S-X)+y X+\bar{W}$, we can also write,

$$
\left.\frac{\partial \bar{W}}{\partial \delta^{k}}=\frac{\bar{\alpha}^{+} \alpha^{k}}{\left(1+\bar{\alpha}^{+}\right)\left(1+\sum_{k^{\prime}=1}^{K} \delta^{k^{\prime}} \alpha^{k^{\prime}}\right)}\left[(1-\Delta) \bar{W}-\Delta\left(1+\sum_{k^{\prime}=1}^{K} \delta^{k^{\prime}} \alpha^{k^{\prime}}\right)(S-X)-y \Delta X\right)\right]
$$

Equations (49) and (50) are used in footnote 10. Substituting $y=0$ in equations (49) and (50), respectively, we get $\partial \bar{W} / \partial \delta^{k}$ of equation (12) and $\partial \bar{W} / \partial \delta^{k}$ of footnote 8 , respectively. 


\section{References}

[1] Black, F. and M. Scholes, 1973, The pricing of options and corporate liabilities, Journal of Political Economy, 81, 637-59.

[2] Bergman, Y. Z., B. D. Grundy and Z. Wiener, 1996, General properties of option prices, Journal of Finance, 51 (5), 1573-1610.

[3] Brennan, M. and E. S. Schwarz, 1977, Convertible bonds: Valuation and optimal strategies for call and conversion. Journal of Finance, 32, 1669-1715.

[4] Constantinides, G., 1984, Warrant exercise and bond conversion in competitive markets, Journal of Financial Economics, 13, 371-97.

[5] Constantinides, G. and R. Rosenthal, 1984, Strategic analysis of the competitive exercise of certain financial options, Journal of Economic Theory, 32, 128-138.

[6] Darsinos, T. and S. Satchell, 2002, On the valuation of warrants and executive stock options: Pricing formulae for firms with multiple warrants/executive options. Working paper, University of Cambridge.

[7] Dennis, J. P. and R. J. Rendleman, 2003, A model for valuing multiple executive stock options issued by the same company, Working paper, University of Virginia.

[8] Emanuel, D., 1983, Warrant valuation and exercise strategy, Journal of Financial Economics, 12, 211-235.

[9] Galai, D. and M. I. Schneller, 1978, Pricing of warrants and the value of the firm, Journal of Finance, 33, 1333-1342.

[10] Grenadier, Steven, 1996, The strategic exercise of options: Development cascades and overbuilding in real estate markets, Journal of Finance, 51, 1653-1679.

[11] Grenadier, Steven, 2002, Option exercise games: An application to the equilibrium strategies of firms, Review of Financial Studies, 15(3), 691-721.

[12] Green, Richard, 1984, Investment incentives, debt and warrants, Journal of Financial Economics 13, 115-136.

[13] Heston, S., 1993, A closed form solution for options with stochastic volatility with applications to bond and currency Options, Review of Financial Studies, 6(2), 327-343. 
[14] Ingersoll, J., 1977, A contingent-claims valuation of convertible securities, Journal of Financial Economics, 4, 289-322.

[15] Ingersoll, J., 1987, Theory of Financial Decision Making, Rowman and Littlefield:NJ.

[16] Mayers, D., 1998. Why firms issue convertible bonds: the matching of financial and real investment options, Journal of Financial Economics 47, 83-102.

[17] Merton, R., 1973, Theory of rational option pricing, Bell Journal of Economics and Management Science, 4 (Spring 1973), 41-83.

[18] Merton, R. (1974). On the pricing of corporate debt: The risk structure of interest Rates, Journal of Finance, v29, 449-470.

[19] Schulz, U. G. and S. Trautmann, 1994, Robustness of option-like warrant valuation, Journal of Banking and Finance (18), 841-859.

[20] Spatt, C.S. and F. P. Sterbenz, 1988, Warrant exercise, dividends and reinvestment policy, Journal of Finance, 43, 493-506.

[21] Stein, J. C., 1992, Convertible bonds as backdoor equity financing, Journal of Financial Economics 32, 3-21.

[22] Williams, J. T., 1993. Equilibrium and options on real assets, Review of Financial Studies, $6,825-850$. 